\title{
Symmetry breaking in photosystem I: ultrafast optical studies of variants near the accessory chlorophylls in the $A$ - and $B$-branches of electron transfer cofactors
}

\author{
Dmitry A. Cherepanov ${ }^{1}\left[\right.$ ( ) Ivan V. Shelaev ${ }^{1} \cdot$ Fedor E. Gostev $^{1} \cdot$ Victor A. Nadtochenko ${ }^{1,2} \cdot$ Wu Xu$^{3}$. \\ John H. Golbeck ${ }^{4,5}$. Alexey Yu. Semenov ${ }^{1,6}$
}

Received: 20 April 2021 / Accepted: 18 August 2021 / Published online: 3 September 2021

(c) The Author(s), under exclusive licence to European Photochemistry Association, European Society for Photobiology 2021

\begin{abstract}
Femtosecond absorption spectroscopy of Photosystem I (PS I) complexes from the cyanobacterium Synechocystis sp. PCC 6803 was carried out on three pairs of complementary amino acid substitutions located near the second pair of chlorophyll molecules $C h l_{2 \mathrm{~A}}$ and $C h l_{2 \mathrm{~B}}$ (also termed $\mathrm{A}_{-1 \mathrm{~A}}$ and $\mathrm{A}_{-1 \mathrm{~B}}$ ). The absorption dynamics at delays of 0.1-500 ps were analyzed by decomposition into discrete decay-associated spectra and continuously distributed exponential components. The multiexponential deconvolution of the absorption changes revealed that the electron transfer reactions in the PsaA-N600M, PsaA-N600H, and PsaA-N600L variants near the $B$-branch of cofactors are similar to those of the wild type, while the PsaB-N582M, PsaB-N582H, and PsaB-N582L variants near the $A$-branch of cofactors cause significant alterations of the photochemical processes, making them heterogeneous and poorly described by a discrete exponential kinetic model. A redistribution of the unpaired electron between the second and the third monomers $C h l_{2 \mathrm{~A}} / C h l_{2 \mathrm{~B}}$ and $C h l_{3 \mathrm{~A}} / C h l_{3 \mathrm{~B}}$ was identified in the time range of 9-20 ps, and the subsequent reduction of $\mathrm{A}_{1}$ was identified in the time range of 24-70 ps. In the PsaAN600L and PsaB-N582H/L variants, the reduction of $A_{1}$ occurred with a decreased quantum yield of charge separation. The decreased quantum yield correlates with a slowing of the phylloquinone $\mathrm{A}_{0} \rightarrow \mathrm{A}_{1}$ reduction, but not with the initial transient spectra measured at the shortest time delay. The results support a branch competition model, where the electron is sheared between $\mathrm{Chl}_{2 \mathrm{~A}}-\mathrm{Chl}_{3 \mathrm{~A}}$ and $\mathrm{Chl}_{2 \mathrm{~B}}-\mathrm{Chl}_{3 \mathrm{~B}}$ cofactors before its transfer to phylloquinone in either $\mathrm{A}_{1 \mathrm{~A}}$ or $\mathrm{A}_{1 \mathrm{~B}}$ sites.
\end{abstract}

Keywords Photosystem I · Primary charge separation · Electron transfer · Femtosecond absorption spectroscopy · Symmetry breaking

Pushing the limits of flash photolysis to unravel the secrets of biological electron and proton transfer- a topical issue in honour of Klaus Brettel.

Dmitry A. Cherepanov

tscherepanov@gmail.com

1 N.N. Semenov Federal Research Center for Chemical Physics, Russian Academy of Sciences, Kosygina St. 4, Moscow 117977, Russian Federation

2 Department of Chemistry, Lomonosov Moscow State University, Leninskiye Gory 1-3, Moscow 119991, Russian Federation

3 Department of Chemistry, University of Louisiana at Lafayette, Lafayette, LA 70504, USA

\section{Introduction}

Photosystem I (PS I) is a photosynthetic pigment-protein complex located in the membranes of cyanobacteria and plants that reduces the low-potential acceptors ferredoxin (or flavodoxin) necessary for the reduction of $\mathrm{NADP}^{+}$. The core

4 Department of Biochemistry and Molecular Biology, The Pennsylvania State University, University Park, PA 16801, USA

5 Department of Chemistry, The Pennsylvania State University, University Park, PA 16801, USA

6 A.N. Belozersky Institute of Physical-Chemical Biology, Lomonosov Moscow State University, Leninskie Gory, 1, Moscow 119992, Russian Federation 
subunits $\mathrm{PsaA}$ and $\mathrm{PsaB}$ harbor the redox cofactors $\mathrm{P}_{700}, \mathrm{~A}_{0}$ and $\mathrm{A}_{1}$, which form symmetrical branches $A$ and $B$ [1-3]. The special pair $\mathrm{P}_{700}$ is a pseudo-dimer of chlorophyll (Chl) $a$ and $a$ molecules located at the pseudo $\mathrm{C}_{2}$-axis of PsaA/ $\mathrm{PsaB}$ heterodimer. The symmetrical branches of redoxactive cofactors are represented by two pairs of chlorophyll molecules $\mathrm{Chl}_{2 \mathrm{~A}} \mathrm{Chl}_{3 \mathrm{~A}}$ and $C h l_{2 B} \mathrm{Chl}_{3 \mathrm{~B}}$, and the phylloquinone molecules $\mathrm{A}_{1 \mathrm{~A}}$ and $\mathrm{A}_{1 \mathrm{~B}}$ (Fig. 1A). The two branches converge at the $[4 \mathrm{Fe} 4 \mathrm{~S}]$ cluster $\mathrm{F}_{\mathrm{X}}$ located at the pseudo $\mathrm{C}_{2}$-axis of the PsaA/PsaB heterodimer. The light-harvesting antenna (LHA) of cyanobacteria contains $90 \mathrm{Chl} a$ and 22 $\beta$-carotene molecules [1].

The mechanism of primary charge separation in PS I is not fully ascertained. According to one of the models, the primary charge separation is initiated by oxidation of the electron donor $\mathrm{P}_{700}$ and reduction one of the two electron acceptors $\mathrm{A}_{0 \mathrm{~A}}$ or $\mathrm{A}_{0 \mathrm{~B}}$, which are formed by the electronically coupled $\mathrm{Chl}_{2 \mathrm{~A}} \mathrm{Chl}_{3 \mathrm{~A}}$ or $\mathrm{Chl}_{2 B} \mathrm{Chl}_{3 \mathrm{~B}}$ pairs [4-6]. In an alternative model, the primary ion-radical states $\mathrm{Chl}_{2 \mathrm{~A}}{ }^{+} \mathrm{Chl}_{3 \mathrm{~A}}{ }^{-}$or $\mathrm{Chl}_{2 \mathrm{~B}}{ }^{+} \mathrm{Chl}_{3 \mathrm{~B}}{ }^{-}$are formed within the $\mathrm{A}_{0 \mathrm{~A}}$ or $\mathrm{A}_{0 \mathrm{~B}}$ pairs in the time range of 6-9 ps and is followed by reduction of $\mathrm{Chl}_{2}{ }^{+}$by $\mathrm{P}_{700}$ as the second step [7-10]. Verification of these hypotheses is challenging, because both the LHA and the $\mathrm{RC}$ are inseparably integrated in a single pigment-protein complex, so that the electron transfer reactions in the RC are in most cases kinetically controlled by energy transfer processes in the time range of a few picoseconds [11]. The results of femtosecond pump/probe spectroscopy with excitation in the far-red region of 720-760 nm revealed the appearance of the charge separated state $\mathrm{P}_{700}{ }^{+} \mathrm{A}_{0}{ }^{-}$as early as $<180 \mathrm{fs}[12,13]$. The basic kinetic scheme of energy and electron transfer reactions in the PS I from Synechocystis sp. PCC 6803 as summarized from the references [8, 12, 14-17] and the present work is shown in Fig. 1B.

The functioning of both $A$ and $B$ branches of the redox cofactors was demonstrated by substitution of Trp residues
PsaA-W693 and PsaB-W673 in the vicinity of $A_{1 A}$ and $A_{1 B}$ by Phe residues, and by Glu residues PsaA-E695 and PsaAE698 with Gln, both in PS I from Chlamydomonas reinhardtii [18]. Replacements of amino acids interacting with the $C h l_{3 \mathrm{~A}}$ and $C h l_{3 \mathrm{~B}}$ monomers were analyzed in PS I from C. reinhardtii $[16,19-24]$ and the cyanobacterium Synechocystis sp. PCC 6803 [25-28]. Substitutions of PsaB-Asn591 and PsaA-Asn604 (numbering according to C. reinhardtii), which ligate water molecules coordinating the magnesium atoms of Chl molecules $C h l_{2 \mathrm{~A}}$ and $C h l_{2 \mathrm{~B}}$, by Leu in the PS I complexes from C. reinhardtii and Synechocystis sp. PCC 6803, were also recently published [29].

The thermodynamically irreversible process on the picosecond timescale is the reduction of phylloquinone in the two symmetrically located sites $\mathrm{A}_{1 \mathrm{~A}}$ and $\mathrm{A}_{1 \mathrm{~B}}$. This forms the secondary ion-radical pairs $\mathrm{P}_{700}{ }^{+} \mathrm{A}_{1 \mathrm{~A}}{ }^{-}$and $\mathrm{P}_{700}{ }^{+} \mathrm{A}_{1 \mathrm{~B}}{ }^{-}$, the stable final photochemical products. The ratio of electron transfer along the $A$ and $B$ branches in cyanobacteria is $\sim 3: 1$ at room temperature [30]. The lifetime of the states $\mathrm{P}_{700}{ }^{+} \mathrm{A}_{1 \mathrm{~A}}{ }^{-}$and $\mathrm{P}_{700}{ }^{+} \mathrm{A}_{1 \mathrm{~B}}{ }^{-}$are $\sim 20 \mathrm{~ns}$ and $\sim 200 \mathrm{~ns}$, after which the electron proceeds forward to the $F_{X}$ cluster (reviewed in [31, 32]). If electron transfer to $F_{X}$ is blocked, the charge recombination reactions $\mathrm{P}_{700}{ }^{+} \mathrm{A}_{1 \mathrm{~A}}{ }^{-} \rightarrow \mathrm{P}_{700} \mathrm{~A}_{1 \mathrm{~A}}$ and $\mathrm{P}_{700}{ }^{+} \mathrm{A}_{1 \mathrm{~B}}{ }^{-} \rightarrow \mathrm{P}_{700} \mathrm{~A}_{1 \mathrm{~B}}$ occur in $\sim 100$ and $\sim 15 \mu$ s, respectively $[30,33,34]$.

Two models of the bidirectional mechanism have been proposed: the "donor-side equilibrium model" and the "branch competition model" [19]. In the first model, two alternative conformations in the RC determine the route of electron transfer along either the $A$ - or $B$-branch; the operation of each branch is independent of the other. In the second model, both branches compete for electrons from $\mathrm{P}_{700}$, hence, a decrease in electron transfer along one of the branches results in an increase of electron transfer along the other branch. Results obtained with different variants do not provide convincing evidence in favor of any particular model. For example, results obtained with
Fig. 1 Two branches of redoxactive cofactors (A) and the main electronic transitions $(\mathbf{B})$ in the RC of PS I. The cofactors include symmetrical pairs of chlorophyll $\mathrm{P}_{\mathrm{A}} / \mathrm{P}_{\mathrm{B}}$ (yellow), $\mathrm{Chl}_{2 A} / \mathrm{Chl_{2B }}$ (orange), $\mathrm{Chl}_{3 A}$ ' $\mathrm{Chl}_{3 B}$ (red), and phylloquinone $\mathrm{A}_{1 \mathrm{~A}} / \mathrm{A}_{1 \mathrm{~B}}$ (green). The PsaAN600 and PsaB-N582 residues contain water molecules (silver spheres) that serve as axial ligands to $C h l_{2 B}$ and $C h l_{2 A}$

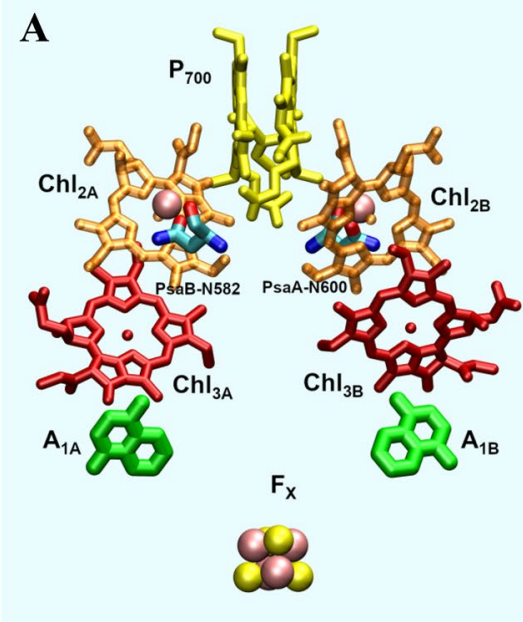

$\lambda$

B

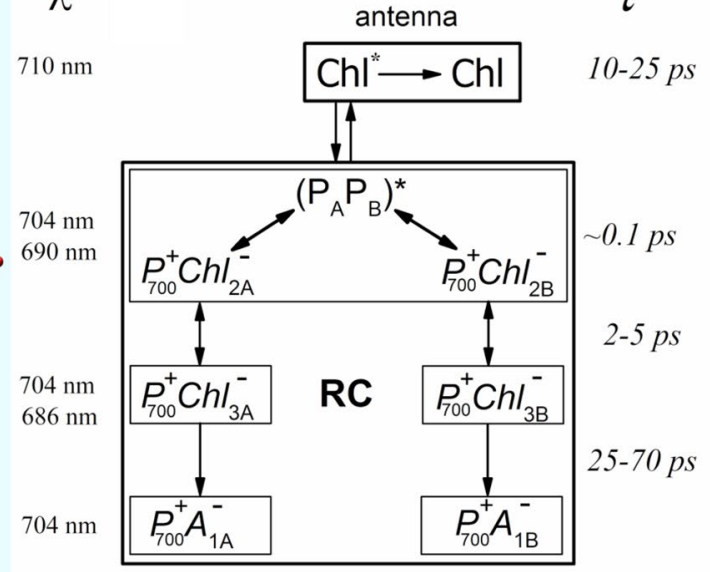


the substitutions near the $\mathrm{Chl}_{3 \mathrm{~A}} / \mathrm{Chl}_{3 \mathrm{~B}}$ cofactors were more consistent with the "branch competition model", where both branches compete for electrons from the primary donor $\mathrm{P}_{700}$. In contrast, the substitutions near the $\mathrm{Chl}_{2 \mathrm{~A}} / \mathrm{Chl}_{2 \mathrm{~B}}$ cofactors were more consistent with the "donor-side equilibrium model", where the ratio between branches $A$ and $B$ is determined by the relative populations of two different conformational states [29]. Thus, the mechanism of charge separation and symmetry breaking in the two branches of redox-active cofactors is not well understood and needs further study.

To investigate the role of the accessory Chls $\left(\mathrm{Chl}_{2 \mathrm{~A}}\right.$ and $C h l_{2 \mathrm{~B}}$ ), three pairs of substitutions of the amino acid residues PsaA-N604 (which ligates the $B$-side accessory $\mathrm{Chl}_{2 \mathrm{~B}}$ through an $\mathrm{H}_{2} \mathrm{O}$ molecule) and PsaB-N591 (which ligates the $A$-side accessory $\mathrm{Chl}_{2 \mathrm{~A}}$ through an $\mathrm{H}_{2} \mathrm{O}$ molecule) with Met, His, and Leu, respectively, were previously studied using time-resolved optical and EPR spectroscopy [29]. Ultrafast transient spectroscopy experiments were performed using laser pulses at $690-700 \mathrm{~nm}$ with a full width at half maximum (FWHM) of 0.15 ps. The global analyses of the transient absorption decay-associated difference spectra revealed in the altered complexes two kinetic components with lifetimes of 1-2 ps and 20-35 ps. The shortest component was ascribed to the redistribution of excitation within the antenna complex, while the slower component was attributed to the energy trapping as evidenced by phylloquinone reduction [29]. In the experiments performed in the present paper, PS I complexes from the same three pairs of mutants were studied using femtosecond pulses centered at $720 \mathrm{~nm}$ with an FWHM of $0.025 \mathrm{ps}$. In agreement with the importance of electron transfer through the $A$-side cofactors, we found that the optical dynamics in PsaA-N600M, PsaA-N600H, PsaAN600L (which affect $B$-side electron transfer) are similar to those of the wild type, while those in PsaB-N582H and PsaB-N582L (which affect $A$-side electron transfer) lead to significant changes in rates of the photochemical reactions.

In a previous paper, we analyzed the primary charge separation in the same PsaA-N600M/H/L and PsaBN582M/H/L variants from cyanobacteria Synechocystis sp. PCC 6803 by focusing on the earliest events in charge separation [35]. We showed that the primary charge separation (lifetime $<100 \mathrm{fs}$ ) proceeds within a symmetric tetrameric exciplex in which the excited state $\left(C h l_{2 \mathrm{~A}} P_{\mathrm{A}} P_{\mathrm{B}} C h l_{2 \mathrm{~B}}\right)^{*}$ is mixed with charge-transfer states $P_{700}{ }^{+} \mathrm{Chl}_{2 \mathrm{~A}}{ }^{-}$and $\mathrm{P}_{700}{ }^{+} \mathrm{Chl}_{2 \mathrm{~B}}{ }^{-}$. In addition, some data on the subsequent electron transfer to the secondary phylloquinone acceptor $A_{1}$ in PS I for six variants were presented. However, the quantum yield and the kinetic analysis of the subsequent formation of the $\mathrm{P}_{700}{ }^{+} \mathrm{A}_{1}{ }^{-}$radical pair formation were not reported. These issues are considered below.

\section{Materials and methods}

\subsection{Sample preparation}

The Synechocystis sp. PCC 6803 recipient strain pWX3 was constructed with deletion of part of the $p s a A$ gene and deletion of the entire $p s a B$ gene for site-directed mutagenesis of the $p s a A$ and $p s a B$ genes as described previously $[28,36]$. For generating site-specific mutations in the psaA gene (PsaA-N600M, PsaA-N600H, PsaA-N600L), the pIBC plasmid was constructed through cloning a DNA fragment containing the majority of $p s a A, p s a B$ and a $760 \mathrm{bp}$ downstream region of the $p s a B$ gene into the pBluescript II KS vector. A chloramphenicol resistant cassette gene was inserted after the $3^{\prime}$ terminator of the $p s a B$ gene. For generating site-specific mutations in the $p s a B$ gene (PsaB-N582M, PsaB-N582H, and PsaB-N582L), the plasmid $\mathrm{pBC}$ was constructed by cloning a 1588-bp of the $p s a B 3^{\prime}$ region and a 760-bp region downstream of the $p s a B$ into the pBluescript II KS vector. A chloramphenicol resistance gene was inserted at the EcoRI site just downstream of $p s a B$ gene. The Synechocystis sp. PCC 6803 recipient strain, pCRT $\Delta B$, was used for site-directed mutagenesis of the $p s a B$ gene. PCR mutagenesis was carried out using the QuikChange site-directed mutagenesis kit (Stratagene, La Jolla, CA). The mutated plasmid constructs that were verified though sequencing were used to transform the Synechocystis sp. PCC 6803 recipient strains. Segregation of the transformants was carried out on screening for chloramphenicol resistance. Full segregation of the desired PsaA-N600M, PsaA-N600H, PsaAN600L and PsaB-N582M, PsaB-N582H, and PsaB-N582L variants was verified through PCR and DNA sequencing. PS I complexes from the wild type and the PsaAN600M, PsaA-N600H, PsaA-N600L and PsaB-N582M, PsaB-N582H, and PsaB-N582L variants were isolated as described previously [29].

\subsection{Ultrafast spectroscopy}

Time-resolved difference absorption spectra $\Delta A(\lambda, t)$ were measured by a pump-probe method described elsewhere [12]. Femtosecond pulses were generated by the Ti:Sapphire laser Tsunami (Spectra-Physics, USA). The laser produced the pulses of duration $\sim 80$ fs at the wavelength $800 \mathrm{~nm}$ with the energy $10 \mathrm{~nJ}$ and repetition rate $80 \mathrm{MHz}$. The Ti:Sapphire laser was pumped by the continuous laser Millennia (Spectra-Physics, USA). A regenerative amplifier system Spitfire (Spectra-Physics, USA) pumped by the solid-state laser Evolution X (Spectra-Physics, USA). The amplified pulses possessed the 
energy of $1 \mathrm{~mJ}$ with the repetition rate of $60 \mathrm{~Hz}$. Thereafter, the pulses were split into pump and probe beams using a dividing mirror. The amplified pulses were split into two beams. One-half of the energy was directed into a noncollinearly phase-matched optical parametric amplifier. Its output centered at $720 \mathrm{~nm}$ with a bandwidth of $\sim 38 \mathrm{~nm}$ (FWHM) was subsequently compressed by a pair of quartz prisms. The gauss pulse of $23 \mathrm{fs}$ was used as a pump. The other half of the energy was focused on a quartz cell with $\mathrm{H}_{2} \mathrm{O}$ to generate supercontinuum probe pulses. The pump and probe pulses were time-delayed with respect to each other by means of a computer-controlled delay stage. They were then attenuated, recombined, and focused on the sample cell with an optical length of the spot of $100 \mu \mathrm{m}$. The pump pulse energy was attenuated to $20 \mathrm{~nJ}$ in all experiments with open PS I (reduced $\mathrm{P}_{700}$ ) and 50 $\mathrm{nJ}$ in the experiments with closed PS I (pre-oxidized $\mathrm{P}_{700}$ ). The pump light spot had a diameter of $200 \mu \mathrm{m}$. The relative polarizations of pump and probe beams were adjusted to 54.7 (magic angle). After the sample, the supercontinuum was dispersed by a polychromator (Acton SP-300) and detected by a CCD camera (Roper Scientific SPEC10). Absorption difference spectra were recorded over the range $400-800 \mathrm{~nm}$. The experiments were carried out at $6{ }^{\circ} \mathrm{C}$ in a 0.5 -mm flow optical cell with optical windows of $0.1-\mathrm{mm}$ thickness. The circulation rate in the flow cell was fast enough to avoid multiple excitations of the same sample volume. The measured spectra were corrected for group delay dispersion of the supercontinuum using a procedure described previously [37, 38].

The incubation mixture contained $50 \mathrm{mM}$ Tris $-\mathrm{HCl}(\mathrm{pH}$ 8.0) and $0.03 \% n$-dodecyl- $\beta$-D-maltoside; the Chl concentration was $0.4 \mathrm{mg} \mathrm{mL}^{-1}$. Experiments with open PS I RCs were performed in the presence of $10 \mathrm{mM}$ sodium ascorbate and $4 \mu \mathrm{M}$ 2,6-dichlorophenolindophenol (DCIP). To prepare closed RCs $\mathrm{P}_{700}$ was oxidized in the absence of ascorbate and DCIP by illumination of the sample by a CW laser with $10 \mathrm{~mW}$ at $532 \mathrm{~nm}$, the light spot being $6 \mathrm{~mm}$ in diameter. The light beam was directed $7^{\circ}$ relative to the pump-probe beams ( $\sim 3^{\circ}$ between the latter).

\subsection{CONTIN analysis of spectral changes}

The spectral changes $\Delta A(\lambda, t)$ were analyzed in the spectral range of $400-780 \mathrm{~nm}$ and in the time interval of $0.05-500$ ps with the program CONTIN [39]. CONTIN is a program invented for the solution of noisy ill-posed linear operator equations, including inverse Laplace transform of relaxation dynamics. At each wavelength $\lambda_{n}$ $(n=1, \ldots, N)$ the spectral changes $Y_{\lambda}(t)=\Delta A\left(\lambda_{n}, t_{m}\right)$ were discretized at the time arrays $\left(t_{1}, \ldots, t_{M}\right)$ of the dimension $M$. A direct inverse Laplace transforms result in a large number of possible solutions with arbitrarily large deviations from each other. CONTIN implements the inverse Laplace transform to deconvolute non-monotonous kinetics $Y_{\lambda}(t)$ into a spectrum of exponential components with the characteristic times $\tau_{k}(k=1, \ldots, K)$ evenly spaced in the logarithmic timescale in combination with Tikhonov-Phillips regularization, which minimizes the sum of the squares of the discretized second derivatives of the solution, resulting in a quasi-continuous spectrum with the local smoothness determined by the regularizing parameter $\alpha$. Namely, for a given relaxation kinetics $Y_{\lambda}(t)$ CONTIN suggests an array of solutions $F_{\nu}(\nu=1, \ldots, \Xi)$ :

$F_{\nu}(t)=\sum_{k} a_{v, k} \cdot \mathrm{e}^{-t / \tau_{k}}$

where $a_{\nu, \mathrm{k}}$ is the amplitude of exponential component $\exp \left(-t / \tau_{k}\right)$ obtained at the given value $\alpha_{\nu}$ of the regularizing parameter. The variance (mean squared error) of solution $v$ is

$\sigma_{v}^{2}=(M-K)^{-1} \sum_{m=1}^{M}\left(Y_{\lambda}\left(t_{m}\right)-\sum_{k=1}^{K} a_{\nu, k} \cdot \mathrm{e}^{-t_{m} / \tau_{k}}\right)^{2}$.

In the absence of prior information regarding the shape of kinetic spectrum, CONTIN suggests an "optimal" solution with the most statistically reliable $\alpha_{\bar{v}}$ value on the basis of an $F$ test. In particular, an assumption on the nonnegative sign of amplitudes $a_{\nu, \mathrm{k}}$ can be imposed for some components $k$. However, this procedure produces spectra, which deviate widely from each other for close $\lambda$ values. To obtain a quasi-continuous behavior of solution against $\lambda$, an additional regularization was applied to select the appropriate $\alpha$ values. Namely, the spectral profile at a given wavelength $\lambda_{n}(n=1, \ldots, N)$ should have minimal deviation from the neighboring spectral profiles. The optimal solution $\nu_{n}$ for optical changes $\Delta A\left(\lambda_{n}, t_{m}\right)$ at the given wavelength $\lambda_{n}$ was found by minimizing the discrepancy:

$D_{n}=\sum_{k=1}^{K}\left(a_{v_{n-1}, k}-2 a_{v_{n}, k}+a_{v_{n+1}, k}\right)^{2}$,

where the range of variation of the parameters $\nu_{n-1}, \nu_{n}$ and $\nu_{n+1}$ was limited by the condition:

$\sigma_{v_{n-1}}^{2}+\sigma_{v_{n}}^{2}+\sigma_{v_{n+1}}^{2} \leq \sigma_{\bar{v}_{n-1}}^{2}+\sigma_{\bar{v}_{n}}^{2}+\sigma_{\bar{v}_{n+1}}^{2}$.

Here, $\sigma_{v_{i}}^{2}$ is the mean squared error of CONTIN solution $\nu_{i}$ for $i$ th kinetic curve discretized at the time-array $\left(t_{1}, \ldots\right.$ ,$t_{\mathrm{M}}$ ) belonging to the wavelength interval $\lambda_{n} ; \boldsymbol{a}_{\boldsymbol{v}_{n}, \boldsymbol{k}}$ is the amplitude of $k$ th exponent with lifetime $\tau_{k}$ of CONTIN solution $\nu_{n}$ for the kinetic curve belonging to the wavelength $\lambda_{n}$. Equation (3) characterizes the smoothness of solutions calculated for neighboring wavelength values $\lambda_{n-1}, \lambda_{n}$, and $\lambda_{n+1}$. 


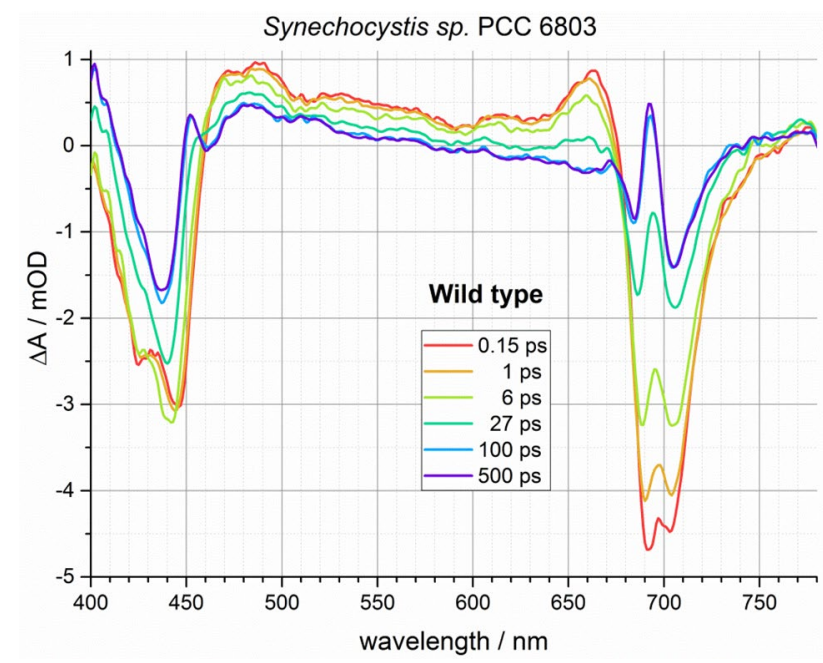

Fig. 2 Transient absorption spectra of wild-type PS I complexes from Synechocystis sp. PCC 6803

\section{Results}

\subsection{Transient absorption changes of photosystem I variants}

The accessory $C h l_{2 \mathrm{~A}}$ and $C h l_{2 \mathrm{~B}}$ monomers in the PS I structure have water as an axial ligand [2], which is not typical for photosynthetic complexes [40]. In PS I from the cyanobacterium Synechocystis sp. PCC 6803 the asparagine residue PsaA-N600 ligates the $B$-side accessory $C h l_{2 \mathrm{~B}}$ through a $\mathrm{H}_{2} \mathrm{O}$ molecule; the PsaB-N582 residue ligates the $A$-side accessory $C h l_{2 \mathrm{~A}}$ similarly. The wild type (WT) and the modified PsaA-N600M/H/L and PsaB-N582M/H/L variants of PS I complexes from the cyanobacterium Synechocystis sp. PCC 6803 were excited by femtosecond pulses centered at $720 \mathrm{~nm}$ (duration 23 fs, FWHM 38 nm, energy 20 nJ). The transient absorption changes of the WT and the six modified PS I variants in the time interval between 0.1 and 500 ps are shown in Figs. 2, 3, 4, and 5; the observed absorption changes arise from a cascade of successive energy and electron transfer processes, which ultimately result in the formation of the secondary ion-radical pair, $\mathrm{P}_{700}{ }^{+} \mathrm{A}_{1}{ }^{-}$, the sole terminating electronic state on the time scale of these measurements. For a qualitative characterization of these processes, the transient spectra were analyzed by their decomposition into discrete exponential components:

$\Delta A(t, \lambda)=\sum_{i=1}^{n} S_{i}(\lambda) \cdot\left[\exp \left(-t / \tau_{i}\right)-1\right]+S_{0}(\lambda)$

Here, $S_{i}(\lambda)$ are the discrete Decay-Associated Spectra (DAS) that arise due to $n$ exponential processes with decay
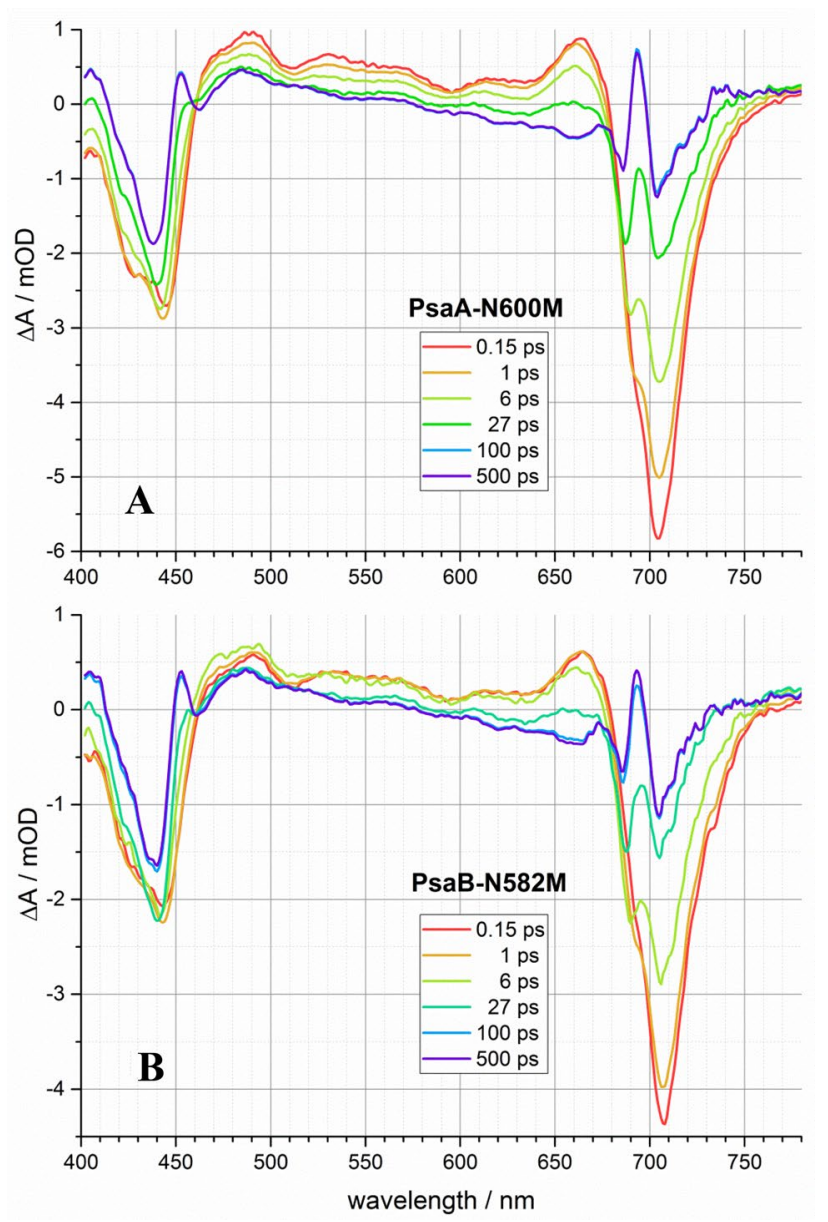

Fig. 3 Transient absorption spectra of PS I complexes from the PsaAN600M (A) and PsaB-N582M (B) variants

times $\tau_{i}$. For a correct description of the overall kinetics of all variants (except PsaA-N600L) four exponential components were required with the decay times summarized in Table 1 (see also Figures S1-S3 of the Supplementary Information). The DAS components may unite diverse electronic transitions occurring in parallel on the same time scale, a factor that complicates their interpretation. Nevertheless, the initial transient spectra $S_{0}(\lambda)$ at the shortest time delay ( $t=100 \mathrm{fs}$ ), and the final spectra $S_{f}(\lambda)=\Delta A\left(\lambda, t_{f}\right)$ at the long time delay ( $t=500 \mathrm{ps}$ ) can be considered as invariants that are insensitive to details of the exponential decomposition in Eq. (5).

When WT PS I is excited at $720 \mathrm{~nm}$, the initial Chl spectrum $S_{0}(\lambda)$ includes two bleach bands in the $\mathrm{Q}_{\mathrm{Y}}$ region with minima at 690 and $704 \mathrm{~nm}$, and two bleach bands in the Soret region with minima at 425 and $445 \mathrm{~nm}$ (Fig. 2, red line). The bleach at $704 \mathrm{~nm}$ represents a mixture of the excited long-wavelength Chl in the LHA [41, 42] and the main band of $\mathrm{P}_{700}$ [43]. The appearance of a bleach at $690 \mathrm{~nm}$ was previously attributed to an ultrafast ( $<100 \mathrm{fs})$ 

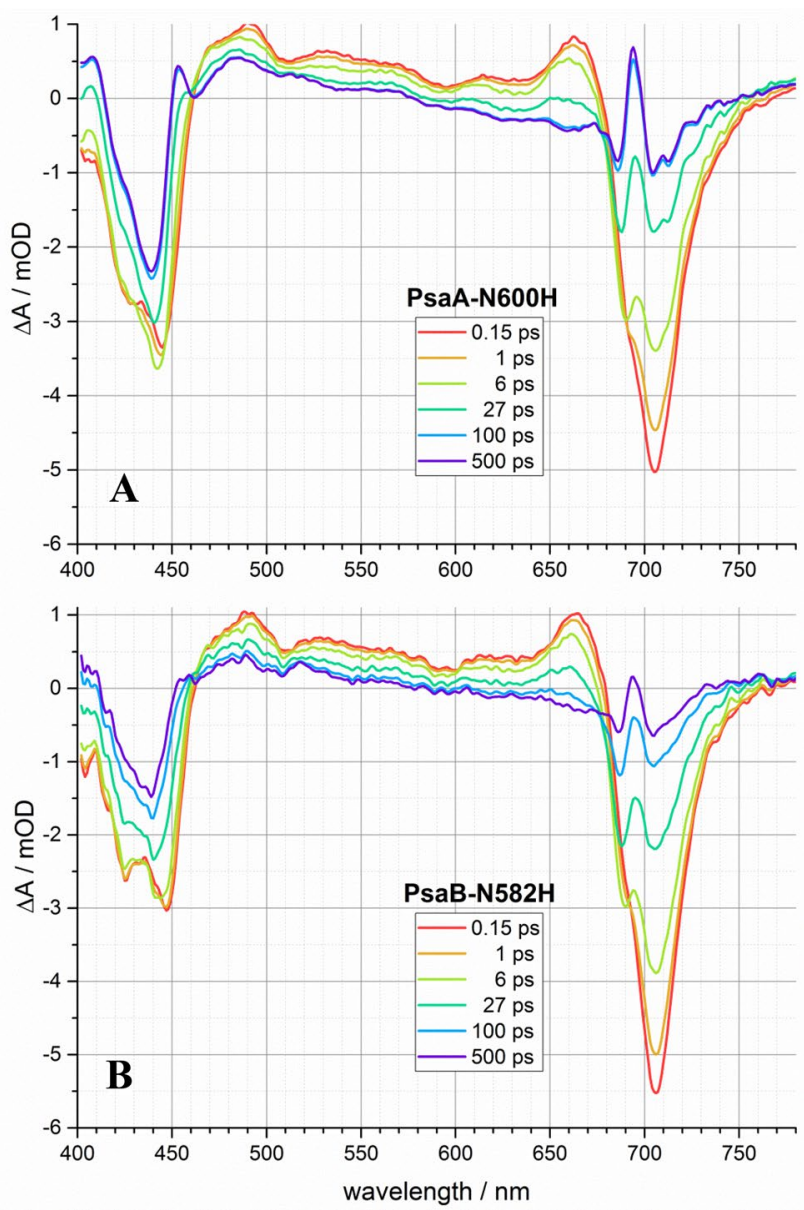

Fig. 4 Transient absorption spectra of PS I complexes from the PsaA$\mathrm{N} 600 \mathrm{H}(\mathbf{A})$ and PsaB-N582H (B) variants

reduction of the primary electron acceptor $\mathrm{A}_{0}$ treated as excitonically coupled dimers $C h l_{2 \mathrm{~A}}-\mathrm{Chl}_{3 \mathrm{~A}}$ and $\mathrm{Chl} l_{2 \mathrm{~B}}-\mathrm{Ch} l_{3 \mathrm{~B}}$ [20] in that fraction of PS I complexes in which the special pair $\mathrm{P}_{700}$ was directly excited in the far-red spectral region $[12,13]$. However, the minimum at $690 \mathrm{~nm}$ observed at the shortest delay is now known to represent the reduced accessory $\mathrm{Chl}_{2 A}$ and $\mathrm{Chl}_{2 B}$ monomers [35], while the electron equilibration between $C h l_{2 \mathrm{~A}} / C h l_{2 \mathrm{~B}}$ and $C h l_{3 \mathrm{~A}} / C h l_{3 \mathrm{~B}}$ proceeds at $2-5$ ps $[10,16,44]$. The excited-state absorption maxima in the region of 650-670 $\mathrm{nm}$ may belong to both the excited state of Chl $a[45,46]$ and to the $\mathrm{A}_{0}$ anion [5].

In the modified PS I variants, the bleach band with a minimum at about $705 \mathrm{~nm}$ predominates in the $\mathrm{Q}_{\mathrm{Y}}$ region of the initial spectra (Figs. 3, 4, 5, red lines). This phenomenon was explained in a recent companion work by a decreased yield of the primary radical ion pairs $P_{700}{ }^{+} \mathrm{Chl}_{2 A}{ }^{-}$or $P_{700}{ }^{+} \mathrm{Chl}_{2 B}{ }^{-}$as a consequence of lowering the redox potential of the respective $C h l_{2 A}$ or $C h l_{2 B}$ monomers due to removal of the $-\mathrm{NH}_{2}$ groups, which directly contact the $C h l_{2 A}$ or $C h l_{2 B}$ macrocycles by hydrogen atoms in the
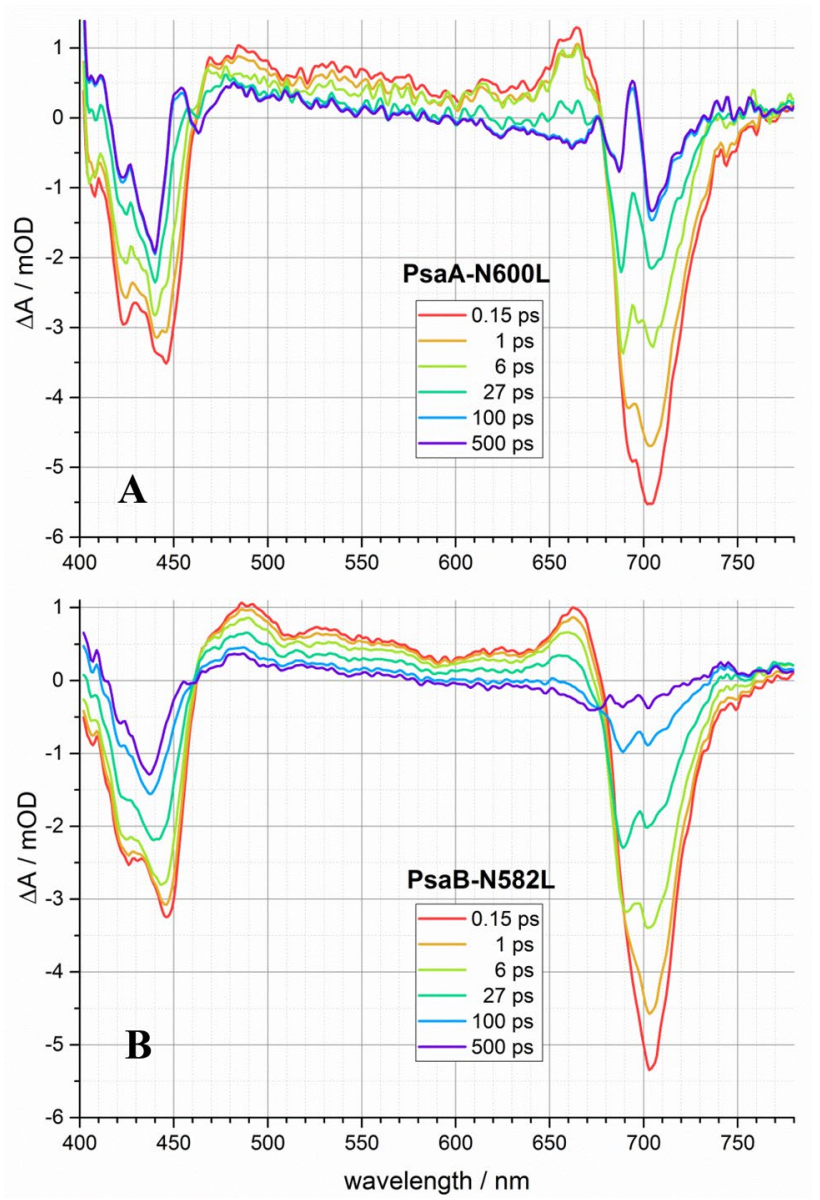

Fig. 5 Transient absorption spectra of PS I complexes from the PsaAN600L (A) and PsaB-N582L (B) variants

wild-type structure [35]. Examination of the data obtained by earlier femtosecond time-resolved absorption and fluorescence spectroscopies gave grounds to conclude that the similar amino acid substitutions lead to blocking of electron transfer in the modified branch of redox cofactors, which was not compensated by electron transfer in the alternative non-modified branch [29]. We reanalyze below the kinetics of energy trapping in the six modified PS I variants and we calculate the quantum yields of the formation of the secondary $\mathrm{P}_{700}{ }^{+} \mathrm{A}_{1}^{-}$ion-radical pairs.

The DAS deconvolution of the absorption dynamics of the WT and the six PS I variants allows us to distinguish four absorption transitions with decay times $\tau_{1}=0.15-0.2 \mathrm{ps}$, $\tau_{2}=1-3 \mathrm{ps}, \tau_{3}=9-18 \mathrm{ps}$, and $\tau_{4}=24-70 \mathrm{ps}$. In all of the six PS I variants, the two fastest transitions $\left(\tau_{1} \approx 0.2\right.$ ps and $\tau_{2}$ $\approx 2 \mathrm{ps}$ ) are accompanied by a notable recovery of absorption at around 700-710 nm (1-2 mOD), a smaller bleaching at $\sim 685 \mathrm{~nm}(0.5-1 \mathrm{mOD})$, and negligible changes in the Soret region at 400-450 nm (Figures S1-S3, red and yellow lines). These fastest processes can be attributed to an uphill energy transfer in the course of excitation equilibration in 
Table 1 Characteristic decay times of absorption changes obtained by global multiexponentiual decomposition

\begin{tabular}{lllll}
\hline Strain & $\tau_{1}(\mathrm{ps})$ & $\tau_{2}(\mathrm{ps})$ & $\tau_{3}(\mathrm{ps})$ & $(\mathrm{ps})$ \\
\hline WT & 0.17 & 1.4 & 8.9 & 24 \\
PsaA-N600M & $0.2^{\mathrm{a}}$ & 1.1 & 12 & 34 \\
PsaB-N582M & & 1.3 & 10 & 31 \\
PsaA-N600H & & 3.1 & 12 & 31 \\
PsaB-N582H & & 2.3 & 19 & 71 \\
PsaA-N600L & & 1.4 & - & 30 \\
PsaB-N582L & & 1.9 & 17 & 69 \\
\hline
\end{tabular}

${ }^{a}$ Decay time was fixed during model optimization

the antenna $[5,47]$ and to the primary charge separation processes in the reaction center [44].

The intermediate transition in the altered variants ( $\tau_{3}=9-18 \mathrm{ps}$ ) is accompanied by a large increase of absorption in the region of $700 \mathrm{~nm}(+2 \mathrm{mOD})$, a small broad bleach in the region of 660-680 $\mathrm{nm}(\sim 0.5 \mathrm{mOD})$, and noticeable multidirectional changes in the Soret absorption area (Figures S1-S3, green lines). Most likely, this transition may be related to the excitation energy transfer from the antenna to the RC, limiting the subsequent formation of the ion-radical states $P_{700}{ }^{+} \mathrm{Chl}_{3 \mathrm{~A}}{ }^{-}$and $P_{700}{ }^{+} \mathrm{Chl}_{3 \mathrm{~B}}{ }^{-}$. This component may also arise due to excitation quenching in the antenna in some of the PS I variants (see below). Because the spectral bands of $\mathrm{Chl}_{2}$ and $\mathrm{Chl}_{3}$ are barely distinguishable in the transient spectra, in the following we apply the term $\mathrm{A}_{0}$ to those electronic states, where the unpaired electron is spread between the $\mathrm{Chl}_{2}$ and $\mathrm{Chl}_{3}$ molecules in both branches and whose $\mathrm{Q}_{\mathrm{Y}}$ absorption bands are positioned near $690 \mathrm{~nm}$. The mixture of these states can be considered as a consolidated primary electron acceptor.

The DAS components of slow transition $\left(\tau_{4}=24-70 \mathrm{ps}\right)$ are characterized by a large increase of absorption in the $\mathrm{Q}_{\mathrm{Y}}$ region (the main peak at $690 \mathrm{~nm}$ and a smaller additional peak at $710 \mathrm{~nm}$ ), a large increase of absorption in the Soret region at 445 and $422 \mathrm{~nm}$, and a moderate decrease of absorption at 650-670 nm (Figures S1-S3, blue lines). This optical transition is certainly related to the reduction of phylloquinone in the $\mathrm{A}_{1 \mathrm{~A}}$ and $\mathrm{A}_{1 \mathrm{~B}}$ sites accompanied by recovering of the $\mathrm{Chl}_{3 \mathrm{~A}}$ and $\mathrm{Chl}_{3 \mathrm{~B}}$ absorption [5, 48-52].

\subsection{Continual multi-exponential analysis of absorption changes}

To visualize the processes of energy migration in the antenna and charge separation in the RC in more detail, the spectral changes in the WT and the PsaA-N600M/H/L and $\mathrm{PsaB}-\mathrm{N} 582 \mathrm{M} / \mathrm{H} / \mathrm{L}$ variants were analyzed in the time interval of $0.1-500$ ps using the CONTIN program, which employs the inverse Laplace transform method for multi-exponential deconvolution of the kinetics [39]. In contrast to the DAS analysis presented above, the CONTIN program determines the decay times of the observed relaxation processes independently at each probe wavelength $\lambda$ and can in principle identify how many processes are observed at different spectral regions. The data obtained are shown in Fig. 6 in the form of two-dimensional spectrograms. Areas in which spectral changes are absent are marked in green, areas with decreased absorption are marked in blue, and areas with increased absorption are marked in red.

The spectrograms of the WT and the modified PsaAN600M/H/L and PsaB-N582M/H/L variants generally confirm the results of the DAS deconvolution in which four optical transitions were identified. The main changes associated with the $\mathrm{A}_{0} \rightarrow \mathrm{A}_{1}$ electron transfer occur at 25-30 ps in the WT, PsaA-N600M, PsaA-N600H, PsaAN600L, and PsaB-N582M variants, but are broadly distributed between 10 and 100 ps in the PsaB-N582H and PsaB-N582L variants. The two latter variants demonstrate a complex behavior, which can be only roughly interpreted in terms of discrete DAS transitions.

Compared to WT PS I, the spectrograms of absorption changes in the altered PS I variants reveal a more extensive process of energy migration from the excited antenna to the $\mathrm{RC}$ at the time range of $1-2 \mathrm{ps}$, which is consistent with the lower yields of the charge transfer state $\mathrm{P}_{700}{ }^{+} \mathrm{Chl}_{2}{ }^{-}$directly generated in the altered PS I variants [35].

The absorption changes in the PsaB-N582M, PsaB$\mathrm{N} 582 \mathrm{H}$, and PsaB-N582L variants include an additional feature that is not seen in the WT and the PsaA-N600M/H/L variants: a prominent absorption increase at $\tau=10-30 \mathrm{ps}$ in the spectral region of 700-720 nm, which is not accompanied by a parallel absorption increase at $690 \mathrm{~nm}$ attributed to the $\mathrm{A}_{0}$ band (white dashed boxes in Fig. $6 \mathrm{f}, \mathrm{g}, \mathrm{h}$ ). This feature can be interpreted as a recovery of the $\mathrm{Chl}$ bleach band in the antenna due to the transition from the excited to the ground state without concomitant energy trapping by the RC. We interpret this transition as an unproductive excitation quenching in $\sim 30 \%$ of the PS I complexes without the formation of charge-separated states in the RC.

Formation of the secondary ion-radical pair $\mathrm{P}_{700}{ }^{+} \mathrm{A}_{1}{ }^{-}$in the PsaB-N582H, and PsaB-N582L variants is seen by a dark-red spot located at $\tau \approx 70$ ps in the narrow spectral range near $690 \mathrm{~nm}$ (Fig. $6 \mathrm{~g}$, h). The slowing of the $\mathrm{A}_{0} \rightarrow \mathrm{A}_{1}$ transition in these PS I variants is also revealed by the DAS analysis (Table 1). In the PsaB-N582H variant, the $\mathrm{A}_{0} \rightarrow \mathrm{A}_{1}$ transition is distinctly distributed between 20 and $80 \mathrm{ps,}$ which may be due to conformational heterogeneity of the PsaB-N582H variant: the $\mathrm{A}_{0} \rightarrow \mathrm{A}_{1}$ electron transfer proceeds by two pathways with substantially different rates, one via the altered and the other via the non-altered redox branches. 
Fig. 6 Continual distribution of the kinetic exponential components of the absorption dynamics in PS I complexes from the wild type (a, e), PsaA-N600M/ PsaB-N582M (b, f), PsaA$\mathrm{N} 600 \mathrm{H} / \mathrm{PsaB}-\mathrm{N} 582 \mathrm{H}(\mathbf{c}, \mathbf{g})$, and PsaA-N600L/PsaB-N582L $(\mathbf{d}, \mathbf{h})$ variants. The scale of the amplitudes of the components is shown to the right of the spectrograms
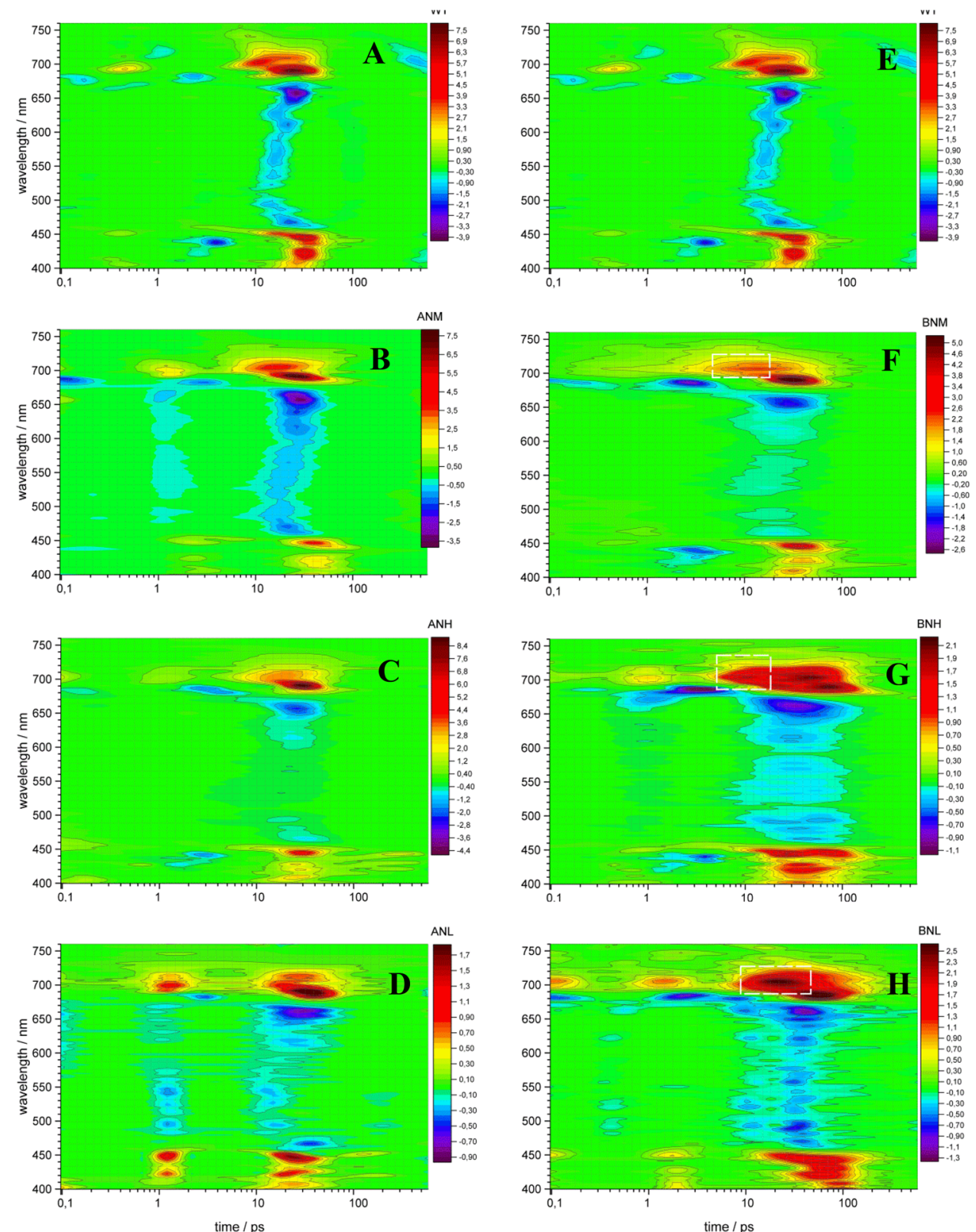

\subsection{Quantum yield of the charge separation reactions}

The quantum yield of the ion-radical state $\mathrm{P}_{700}{ }^{+} \mathrm{A}_{1}{ }^{-}$formed in the PS I variants at the time scale of 500 ps was estimated by comparing the amplitudes of the initial $S_{0}(\lambda)$ and the final $S_{f}(\lambda)$ transient spectra of the WT and the PS I variants. The initial $S_{0}(\lambda)$ spectrum arising at 100 fs upon excitation of PS I in the far-red region represents a mixture of excited Chls of the LHA [41, 42], excited Chls of the special pair $\mathrm{P}_{700}$ [43], and various charge-transfer $\mathrm{Chl}^{+\delta} \mathrm{Chl}^{-\delta}$ states generated both in the LHA and the RC [44]. Because the linear absorption spectra of the PsaA-N600M/H/L and PsaB$\mathrm{N} 582 \mathrm{M} / \mathrm{H} / \mathrm{L}$ variants in the far-red region are very similar to the spectrum of WT PS I [35], significant differences in the $S_{0}(\lambda)$ of the altered PS I complexes in the $\mathrm{Q}_{\mathrm{Y}}$ region (Figs. 2, $3,4,5$, red) indicate changes in the primary photochemical processes as a result of the amino acid substitutions. At the same time, the final $S_{f}(\lambda)$ spectra of all PS I variants (Figs. 2, $3,4,5$, violet) are much less variable.

Figure 7a compares the final $S_{f}(\lambda)$ spectra of the PsaAN600M/H/L PS I variants with that of the WT, and Fig. 7d shows the analogous $S_{f}(\lambda)$ spectra of the PsaB-N582M/H/L PS I variants. These spectra can be unequivocally assigned to the ion-radical state $\mathrm{P}_{700}{ }^{+} \mathrm{A}_{1}{ }^{-}$, where the distribution of electron between phylloquinone in the A- and B-branches remains unidentifiable. In Fig. 7, the spectra of altered PS I complexes are scaled in such a way as to achieve the 
Fig. 7 Superposition of the transient spectra assigned to the secondary ion-radical $\mathrm{P}_{700}{ }^{+} \mathrm{A}_{1}{ }^{-}$pair (a, d), the primary ion-radical $\mathrm{P}_{700}{ }^{+} \mathrm{A}_{0}^{-}$pair $(\mathbf{b}, \mathbf{e})$, and the initial mixed $[$ Ant/RC]* excited state $(\mathbf{c}, \mathbf{f})$ of the PsaA-N600M/H/L (left) and $\mathrm{PsaB}-\mathrm{N} 582 \mathrm{M} / \mathrm{H} / \mathrm{L}$ (right) variants. The transient spectra of the altered PS I variants (colored) were normalized in the $410-450 \mathrm{~nm}$ region to the absorption of the WT (black) by Eq. (6) using the transient spectra of the $\mathrm{P}_{700}{ }^{+} \mathrm{A}_{1}{ }^{-}$ion-radical pairs (a, d)
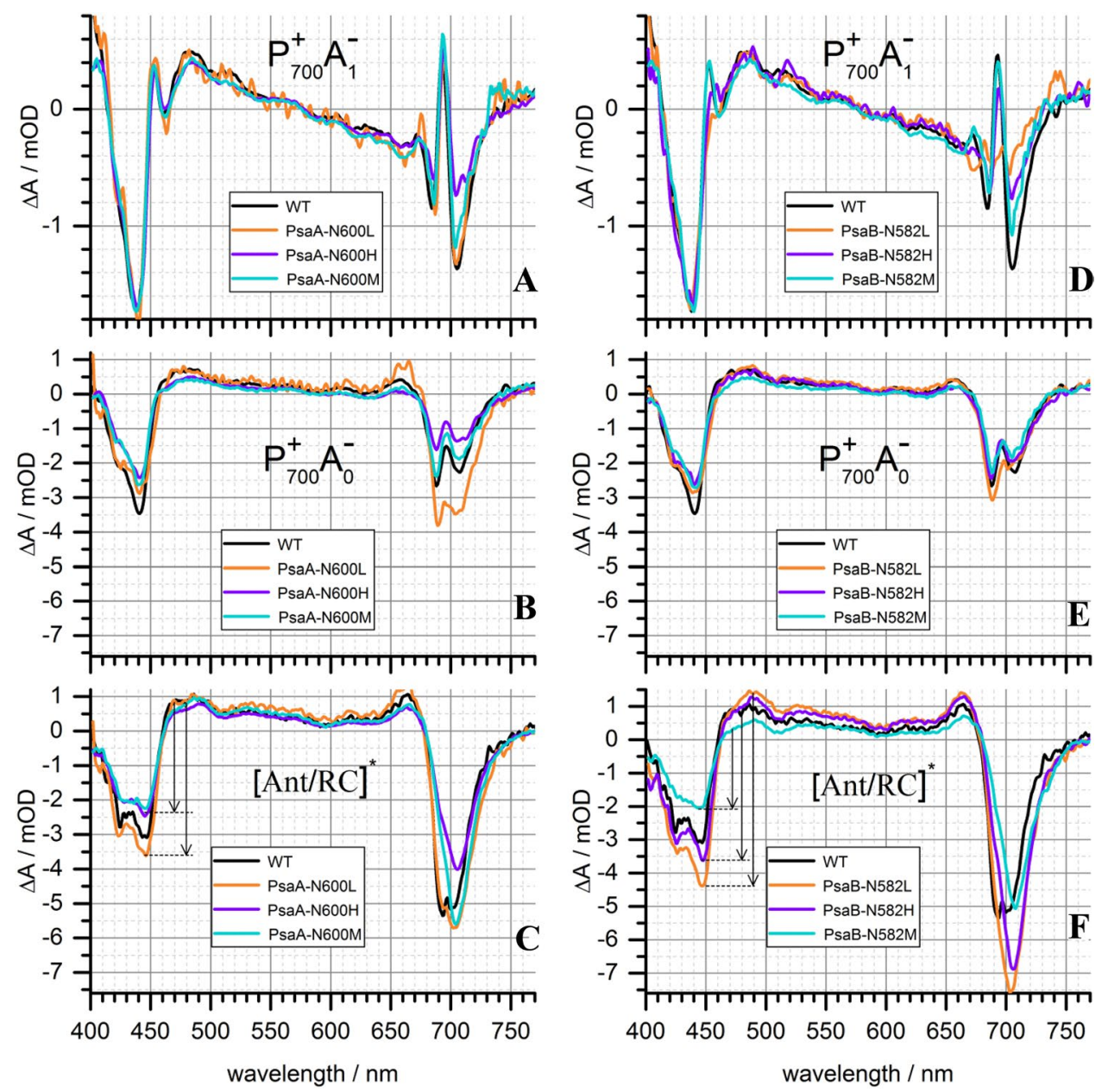

maximal overlap between the altered $S_{f}(\lambda)$ spectrum and that of the WT in the Soret region. The scaling factor $\varepsilon$ was determined for each of the altered complexes by minimizing the residual function:

$y(\varepsilon)=\int_{410}^{450}\left(S_{f}^{w t}(\lambda)-\varepsilon \cdot S_{f}^{m u t}(\lambda)\right)^{2} d \lambda$

which characterizes the deviation of two spectra in the spectral region between 410 and $450 \mathrm{~nm}$. Figure 7 demonstrates that the spectral properties of the PS I core in the Soret region were slightly changed as a result of the amino acid substitutions.

Figure 7 shows also the transient spectra of the $\mathrm{P}_{700}{ }^{+} \mathrm{A}_{0}{ }^{-}$ion-radical states for the PsaA-N600M/H/L (b) and PsaB-N582M/H/L (e) variants, which were calculated under the assumption that the slow DAS transition $\left(\tau_{4}=24-70 \mathrm{ps}\right)$ is related to $A_{0} \rightarrow A_{1}$ electron transfer. It is noteworthy that the spectra of all six PS I variants quantitatively coincide in the Soret region, and five of them (except PsaA-N600L) coincide also in the $\mathrm{Q}_{\mathrm{Y}}$ region.
As a final point, Fig. 7 compares the initial $S_{0}(\lambda)$ spectra of the PsaA-N600M/H/L (c) and PsaB-N582M/H/L (f) variants, which reveal significant differences in the amplitude in the Soret region (indicated by arrows). The amplitude of the Soret band bleach in the PsaA-N600L $S_{0}(\lambda)$ spectrum (c, yellow) is enhanced by a factor of $\sim 1.6$ compared to the PsaA-N600M (green) and the PsaA-N600H (blue) variants. The amplitude of the Soret band bleach in the $S_{0}(\lambda)$ spectrum of PsaB-N582M (f, green) is similar to that of the PsaAN600M and PsaA-N600H variants, but the spectra of PsaB$\mathrm{N} 582 \mathrm{H}$ (f, blue) and PsaB-N582L (f, yellow) are enhanced by factors of $\sim 1.6$ and $\sim 2.4$, respectively.

The differences in the $S_{0}(\lambda)$ spectra may be only partially explained by a varying contribution of the $\mathrm{P}_{700}{ }^{+} \mathrm{Chl}_{2}{ }^{-}$chargetransfer state generated in the symmetric $C h l_{2 A} P_{A} P_{B} C h l_{2 B}$ exciplex upon excitation. This contribution, denoted as the parameter $\alpha$, was calculated by the principal component analysis of the spectra [35] The $\mathrm{P}_{700}{ }^{+} \mathrm{Chl}_{2}{ }^{-}$contribution was maximal in the WT $(\alpha=0.71)$ and minimal in the PsaA-N600M $(\alpha=0.33)$ and PsaB-N582M $(\alpha=0.21)$ variants, which may explain a higher amplitude of the Soret bleach in the WT complex (black) compared to the PsaA-N600M and PsaB-N582M 
variants (green). However, even though the $P_{700}{ }^{+} \mathrm{Chl}_{2}{ }^{-}$contribution was minimal in the PsaB-N582H variant $(\alpha=0.15)$, the Soret bleach amplitude of this complex (f, blue) was greater than that of the WT PS I.

Thus, we interpret the enhanced relative amplitude of the Soret bleach in the initial $S_{0}(\lambda)$ spectra of the PsaA-N600L, PsaB-N582H and PsaB-N582L variants (or equally, the reduced relative amplitude of the Soret bleach in the final $S_{f}(\lambda)$ spectra) as a result of the unproductive excitation quenching in $36-60 \%$ of the PS I complexes on the time scale of 20 ps without the formation of charge-separated states in the RC. The quantum yield of the $\mathrm{P}_{700}{ }^{+} \mathrm{A}_{1}{ }^{-}$formation in the PsaAN600L, PsaB-N582H and PsaB-N582L variants was estimated as $0.64,0.64$, and 0.4 , respectively.

\subsection{Productive and unproductive excitation quenching in open and closed PS I complexes}

In the PS I complexes from Synechocystis sp. PCC 6803 in which the special pair was pre-oxidized to $\mathrm{P}_{700}{ }^{+}$(closed centers), efficient quenching of excited LHA occurred with apparent decay times of 6.5 and 24 ps [53]. Similar trapping times were determined for PS I complexes with open and closed reaction centers by picosecond fluorescence measurements $[14,54]$. The unproductive excitation quenching in the PsaA-N600L, PsaB-N582H and PsaB-N582L variants occurs on the same time scale and may be related to quenching by closed PS I complexes. Figure 8 compares the absorbance changes of WT PS I in the "open" and "closed" states with those of the PsaB-N582L variant in three representative spectral regions: the Soret band $(440 \mathrm{~nm})$, and the blue $(685 \mathrm{~nm})$ and red $(710 \mathrm{~nm})$ edges of the $\mathrm{Chl} \mathrm{Q}_{\mathrm{Y}}$ absorption band. In accordance with previous observations [53], the absorption changes of PS I in the closed state (dotted lines) occurred in the same time range of 5-20 ps, where the excitation trapping by the open centers takes place (solid limes). The kinetics of the PsaB-N582L variant in the open state (dash-dotted lines) include an extensive absorbance recovery at 440 and $710 \mathrm{~nm}$ in the time range of $10 \mathrm{ps}$ (thick arrows), but not at $685 \mathrm{~nm}$ (thin arrow). This kinetic component was observed mainly in the PsaB-N582H and PsaB-N582L variants, where the charge separation is hindered (Table 1). It may be tentatively attributed to the same quenching process as operating in the closed PS I complexes (see Sect. 4.3 of Discussion).

\section{Discussion}

\subsection{Kinetic effects of amino acid substitutions near the $\mathrm{ChI}_{2 \mathrm{~A}}$ and $\mathrm{Chl}_{2 \mathrm{~B}}$ monomers}

Femtosecond measurements of PS I complexes isolated from strains with the same substitutions of PsaA-N600
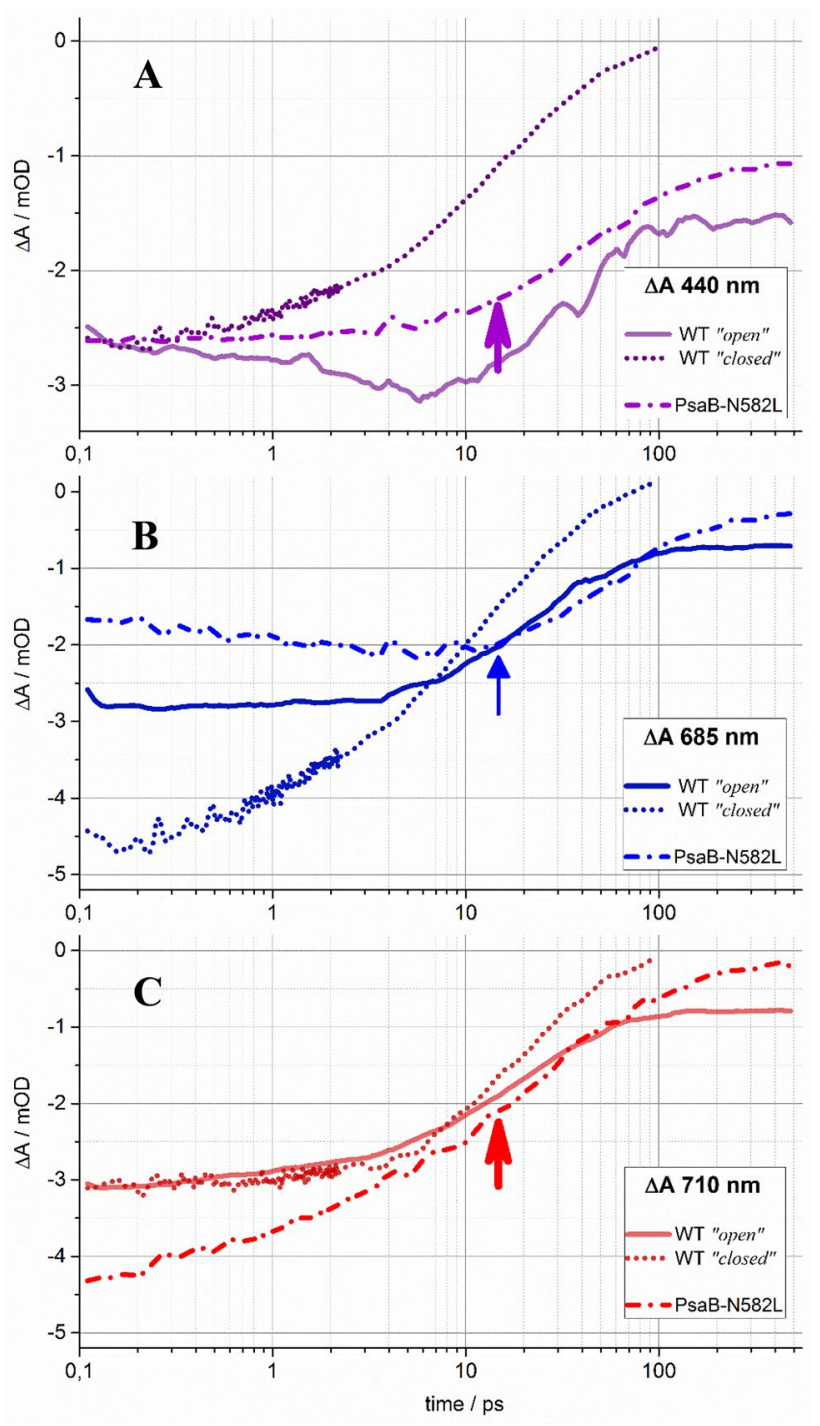

Fig. 8 Absorbance dynamics of the PS I complexes isolated from the WT and the PsaB-N582L variant at 440 (A), 685 (B), and $710 \mathrm{~nm}$ (C). The kinetics of the WT (solid) and the PsaB-N582L variant (dash-dotted) were measured in the presence of ascorbate and DCIP; the PS I in "closed" state (dotted) was measured with the $\mathrm{P}_{700}$ preoxidized by laser excitation at $532 \mathrm{~nm}$. The pump pulses were centered at $720 \mathrm{~nm}$. The thick arrows indicate absorption recovery of the PsaB-N582L variant at 440 and $710 \mathrm{~nm}$ attributed to the nonphotochemical quenching of excited $\mathrm{Chl}$ in the antenna, which is not accompanied by absorption changes at $685 \mathrm{~nm}$ (thin arrow)

and PsaB-N582 by Leu, His, and Met have been published recently by Badshah et al. [29]. The transient absorption changes in the region of $604-750 \mathrm{~nm}$ were analyzed by decomposition to the sum of two exponential decay components and a long-lived offset. Because the excitation in these experiments was centered at $690-700 \mathrm{~nm}$, the fastest component with the decay time of 1-2 ps was attributed to the processes of energy redistribution within the lightharvesting antenna not related to electron transfer reactions 
[29]. The slow component with the decay time of 21-35 ps was attributed to the process of energy trapping owing to $\mathrm{A}_{0} \rightarrow \mathrm{A}_{1}$ electron transfer.

In the present paper, femtosecond measurements were performed in a wide spectral range of $400-800 \mathrm{~nm}$ (Figs. 2, $3,4,5$ ), which allowed us to normalize the difference spectra in the Soret band (Fig. 7) and to calculate thereby the quantum yield of electron transfer in the substituted PS I complexes. Although the Soret region is rarely used in femtosecond studies of PS I, in some cases absorption changes in the blue region provide unique possibilities to resolve electronic transitions indistinguishable in the red spectral range $[55,56]$. Pumping at $720 \mathrm{~nm}$ resulted in direct excitation of the special pair $\mathrm{P}_{700}$ with a moderate contribution of excited antenna chlorophyll [35]. The discrete DAS decomposition of the absorption dynamics in Eq. (5) revealed four transitions with decay times $\tau_{1}=0.15-0.2 \mathrm{ps}, \tau_{2}=1-3 \mathrm{ps}$, $\tau_{3}=9-20 \mathrm{ps}$, and $\tau_{4}=24-70 \mathrm{ps}$ and an offset related to the difference spectrum of the long-lived $\mathrm{P}_{700}{ }^{+} \mathrm{A}_{1}{ }^{-}$ion-radical state (Figures S1-S3). To reveal additional features in the kinetics, continual multi-exponential analysis of the absorption dynamics based on the inverse Laplace transform method was applied (Fig. 6). Because the continual distribution of relaxation processes was calculated independently at each probe wavelength, this method is not contingent on a priori assumptions about the form of the kinetic model of the system under study. The absorption changes on the time scale of $0.2-2 \mathrm{ps}$ are related to the energy redistribution in the antenna $[10,11,53]$ and the primary charge separation in the reaction center $[8,14,15,44,57]$. The slow components with the decay times of 10-70 ps may be related both to the $A_{0} \rightarrow A_{1}$ electron transfer $[51,52]$ and to the excitation quenching observed in the "closed" reaction centers with pre-oxidized $\mathrm{P}_{700}[10,14,53]$.

The $A_{0} \rightarrow A_{1}$ electron transfer, which is associated with the slowest DAS component, is characterized in PS I from Synechocystis sp. PCC 6803 by the recovery of a sharp bleach centered at $\sim 686 \mathrm{~nm}[51,58]$. In the PsaA-N600M, PsaA-N600H, PsaA-N600L, and PsaB-N582M variants the decay time of the $\mathrm{A}_{0} \rightarrow \mathrm{A}_{1}$ transition increased by a factor of 1.3 from 24 ps in the WT to 30-34 ps (Table 1). In the PsaBN582L variant with the altered more active $A$-branch, the kinetics of $\mathrm{A}_{0} \rightarrow \mathrm{A}_{1}$ electron transfer was retarded by a factor of 2.9 and also the difference spectrum of the final ion-radical state $\mathrm{P}_{700}{ }^{+} \mathrm{A}_{1}{ }^{-}$was substantially affected (Fig. 5b). The similar retardation of the $A_{0} \rightarrow A_{1}$ transition was observed in the PsaA-N582H variant, but the $\mathrm{P}_{700}{ }^{+} \mathrm{A}_{1}{ }^{-}$difference spectrum of this strain resembled that of the WT (Fig. 4b). The CONTIN deconvolution of the transient absorption dynamics revealed significant kinetic heterogeneity of the PsaB$\mathrm{N} 582 \mathrm{H}$ and PsaB-N582L variants, where recovery of the bleach at $\sim 710 \mathrm{~nm}$ was attributed to $\mathrm{Chl}^{*}$ quenching in the light-harvesting antenna (marked by white boxes in Fig. 6).
The significant slowing of the $\mathrm{A}_{0} \rightarrow \mathrm{A}_{1}$ transfer as a result of the PsaB-N582H and PsaB-N582L substitutions near the $\mathrm{Chl}_{2 \mathrm{~A}}$ monomer in the A-branch may be related to the asymmetry in the functional activity of the two branches. Various experiments have revealed the predominance of branch $A$ over branch $B$ in a ratio of approx. 3:1 in cyanobacteria [18, $19,30]$. The slowing may be due to a number of factors. First, such a deceleration could occur if the rate constant of $\mathrm{A}_{1 \mathrm{~B}}$ reduction in the $\mathrm{B}$-branch is sufficiently smaller than the rate constant of $A_{1 A}$ reduction in the A-branch (see Fig. 1). However, the data on amino acid substitutions of the ligands to the $\mathrm{Chl}_{3 \mathrm{~A}}$ and $\mathrm{Chl}_{3 \mathrm{~B}}$ monomers indicate that the rate constants of $\mathrm{A}_{1 \mathrm{~A}}$ and $\mathrm{A}_{1 \mathrm{~B}}$ reduction do not differ [23, 28]. Second, a slowdown may take place if the PsaB-N582H and PsaB-N582L substitutions hinder the $\mathrm{Chl}_{2 \mathrm{~A}} \rightarrow \mathrm{Chl}_{3 \mathrm{~A}}$ electron transfer, for example, as a result of a significant increase in the $\mathrm{Chl}_{2 \mathrm{~A}}$ redox potential. However, an analysis of the structural features of the $\mathrm{Chl}_{2 \mathrm{~A}}$ and $\mathrm{Chl}_{2 \mathrm{~B}}$ binding sites showed that these substitutions most probably cause a decrease in the redox potential [35]. For this reason, a plausible rationale for the deceleration of the observed $\mathrm{A}_{0 \mathrm{~A}} \rightarrow \mathrm{A}_{1 \mathrm{~A}}$ transfer is a decrease of the equilibrium constant $K_{23}$ and shift of equilibrium between the excited antenna and the primary ion-radical pair $\mathrm{P}_{700}{ }^{+} \mathrm{A}_{0}{ }^{-}$to the left:

$A n t^{*} \stackrel{K_{12}}{\longleftrightarrow} R C^{*} \stackrel{K_{23}}{\longleftrightarrow} P_{700}^{+} A_{0 A}^{-} \stackrel{k_{34}}{\longrightarrow} P_{700}^{+} A_{1 A}^{-}$.

In this case, the observed rate of $\mathrm{A}_{0 \mathrm{~A}} \rightarrow \mathrm{A}_{1 \mathrm{~A}}$ transfer $V=K_{12} K_{23}\left(1+K_{12}+K_{12} \cdot K_{23}\right)^{-1} k_{34}$ would be appropriately decelerated.

\subsection{Quantum yield and conformational heterogeneity}

The spectral measurements in the work by Badshah et al. [29] were restricted by the red region of $604-750 \mathrm{~nm}$, so the quantum yield of the $\mathrm{P}_{700}{ }^{+} \mathrm{A}_{1}{ }^{-}$formation was estimated by the $\mathrm{P}_{700}$ bleach at $703 \mathrm{~nm}$. The use of a wide spectral interval of 400-800 nm allowed us to normalize the transient spectra using the Soret band and to calculate the quantum yield of electron transfer in the substituted complexes, despite strong changes in the $\mathrm{Q}_{\mathrm{Y}}$ band of the $\mathrm{P}_{700}$ spectrum in the PsaAN600L variant (Fig. 5c, dark blue).

The quantum yields of charge separation in the altered PS I complexes were estimated by comparing the Soret bleach amplitudes in the transient spectra at 0.1 ps delay after their normalization by the absorption at $500 \mathrm{ps}$, i.e., by the spectra attributed to the $\mathrm{P}_{700}{ }^{+} \mathrm{A}_{1}{ }^{-}$state (Fig. 7). The bleaching in the Soret region at the shortest delay is caused by a mixture of three different electronic states: the excited Chls of the antenna, the excited special pair $\mathrm{P}_{700}{ }^{*}$, and the primary ion-radical pair $\mathrm{P}_{700}{ }^{+} \mathrm{Chl}_{2}{ }^{-}$formed in the symmetric $\mathrm{Chl}_{2 A} P_{A} P_{B} C h l_{2 B}$ exciplex [35]. These states 
Table 2 Quantum yields of the secondary $\mathrm{P}_{700}{ }^{+} \mathrm{A}_{1}{ }^{-}$ion-radical pair formation in six PS I variants determined by absorption changes in three different spectral regions

\begin{tabular}{llll}
\hline & Q.Y. (this work) & \multicolumn{2}{l}{ Q.Y. (Badshah et al. } \\
& & \multicolumn{2}{l}{$2018)$} \\
\cline { 3 - 4 } & $\Delta \mathrm{A}_{440}$ & $\mathrm{~A}_{820}$ & $\Delta \mathrm{A}_{703}$ \\
\hline PsaA-N600M & 1 & & \\
PsaB-N582M & 1 & $0.66^{\mathrm{a}}$ & $0.82^{\mathrm{a}}$ \\
PsaA-N600H & 1 & $0.52^{\mathrm{a}}$ & $0.44^{\mathrm{a}}$ \\
PsaB-N582H & 0.64 & 0.71 & 0.87 \\
PsaA-N600L & 0.64 & 0.49 & 0.41 \\
PsaB-N582L & 0.40 & 0
\end{tabular}

${ }^{\text {a }}$ These values appeared in [29] in Supplemental Materials

obey a different molar stoichiometry of ground state depletion: one for the excited $\mathrm{Chl}$ and $\mathrm{P}_{700}$, and two for the ionradical pair $\mathrm{P}_{700}{ }^{+} \mathrm{Chl}_{2}^{-}$. Because the contribution of the $P_{700}{ }^{+} \mathrm{Chl}_{2}{ }^{-}$state to the initial absorption is relatively small in the altered PS I variants [35], the enhanced amplitude of the Soret band bleach in the initial spectra of PsaAN600L (Fig. 6c), PsaB-N582H and PsaB-N582L variants (Fig. 6f) gave the following estimates for the quantum yield of the $\mathrm{P}_{700}{ }^{+} \mathrm{A}_{0}{ }^{-}$and $\mathrm{P}_{700}{ }^{+} \mathrm{A}_{1}{ }^{-}$production: PsaA-N600L (Q.Y.=0.64), PsaB-N582H (Q.Y.=0.64), and PsaB-N582L $(\mathrm{Q} . Y .=0.4)$; in the other three variants the quantum yield is about 1.0 (Table 2). Because the formation of an ion-radical pair from mono-exciton $\left(P_{700} \mathrm{Chl}_{2}\right)^{*} \rightarrow \mathrm{P}_{700}{ }^{+} \mathrm{Chl}_{2}{ }^{-}$is accompanied by the disappearance of two chlorophyll molecules from the ground state, it causes an additional bleaching of the Soret band [59], which is clearly seen in the kinetic trace at $440 \mathrm{~nm}$ (Fig. 8A, solid). For that reason the higher amplitude of the Soret band bleach in the initial spectra of the WT may be caused by a higher extent of the ion-radical pair $\mathrm{P}_{700}{ }^{+} \mathrm{Chl}_{2}{ }^{-}$formation revealed in this variant [35].

The amino acid substitutions near the $\mathrm{Chl}_{2 \mathrm{~A}}$ monomer, particularly the PsaB-N582H and PsaB-N582L variants, revealed a substantial kinetic heterogeneity in the continuum decay decompositions (Fig. 6f-h). It may arise if (i) the redox transitions in the $A$-branch were substantially altered by the substitutions and (ii) the $A$ - and $B$-branches operate independently of each other. However, the last prerequisite is apparently not fulfilled. Byrdin et al. studied the effects of a series of mutations in the $\mathrm{Chl}_{3 \mathrm{~A}} / \mathrm{Chl}_{3 \mathrm{~B}}$ and the $\mathrm{A}_{1 \mathrm{~A}} / \mathrm{A}_{1 \mathrm{~B}}$ binding sites on the kinetics of the phylloquinone reoxidation $[60,61]$. It was possible to resolve both the lifetimes and amplitudes of the $\mathrm{A}_{1 \mathrm{~A}} \rightarrow \mathrm{F}_{\mathrm{X}}$ and $\mathrm{A}_{1 \mathrm{~B}} \rightarrow \mathrm{F}_{\mathrm{X}}$ transitions in WT and in the modified PS I variants. The mutations in the $A_{1 A}$ and $\mathrm{A}_{1 \mathrm{~B}}$ sites slowed the $\mathrm{A}_{1 \mathrm{~A}} \rightarrow \mathrm{F}_{\mathrm{X}}$ and $\mathrm{A}_{1 \mathrm{~B}} \rightarrow \mathrm{F}_{\mathrm{X}}$ transitions, respectively, without changing their amplitudes, while the mutations in the $C h l_{3 \mathrm{~A}}$ and $C h l_{3 \mathrm{~B}}$ sites affected only their amplitudes without changing the rates. These experiments

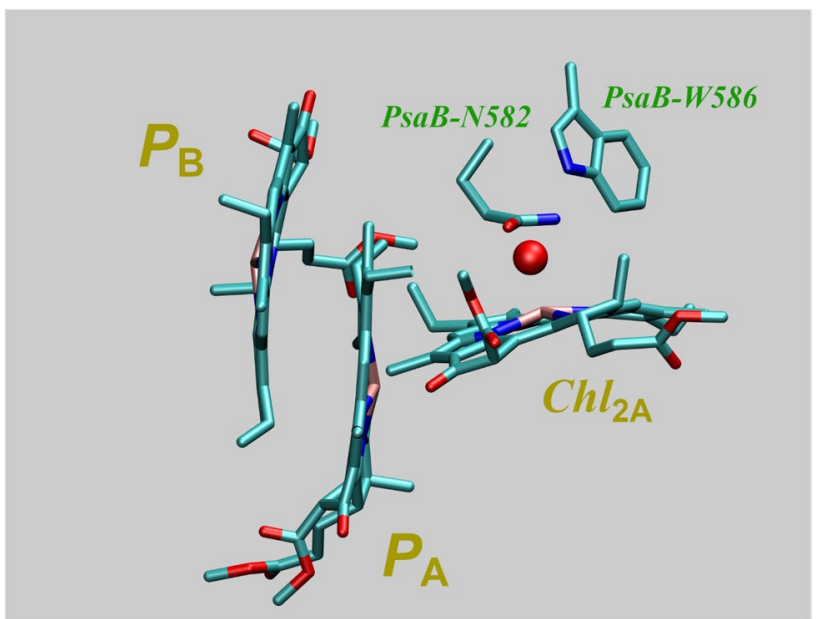

Fig. 9 Out-of-plane deformations of the porphyrin macrocycles in the PS I reaction center from Synechocystis sp. PCC 6803 (pdb entry 5oy0) [2]. The conjugated monomers $\mathrm{P}_{\mathrm{A}}$ and $\mathrm{P}_{\mathrm{B}}$ of the special pair $\mathrm{P}_{700}$ and the adjacent accessory $C h l_{2 \mathrm{~A}}$ in the A-branch of redox cofactors are shown without phytol tails. The residues PsaB-N582 and PsaB-W586 coordinate a water molecule (red sphere), which is an axial ligand of $\mathrm{Chl}_{2 \mathrm{~A}}$

demonstrate that after photoexcitation of the reaction center, a redistribution of the electron density between the chlorophyll dimers $C h l_{2 \mathrm{~A}}-C h l_{3 \mathrm{~A}}$ and $C h l_{2 \mathrm{~B}}-C h l_{3 \mathrm{~B}}$ in the $A$ - and $B$-branches takes place (reversible reactions in Fig. 1B), and only the subsequent reduction of phylloquinone leads to thermodynamically irreversible localization of the electron in one of the $\mathrm{A}_{1 \mathrm{~A}}$ or $\mathrm{A}_{1 \mathrm{~B}}$ sites (irreversible reactions in Fig. 1B). In this regard, the cause of the kinetic dispersion is most likely structural heterogeneity of the PS I variants.

More significant effects of the amino acid substitutions near the $\mathrm{Chl}_{2 \mathrm{~A}}$ monomer may be explained by the more rigid system of $\mathrm{H}$-bonds in the $C h l_{2 \mathrm{~A}}$ site, in view of the fact that the almost strict $\mathrm{C}_{2}$-symmetry is broken in the $\mathrm{Chl}_{2 \mathrm{~A}} / \mathrm{Chl}_{2 \mathrm{~B}}$ binding regions. Namely, in the X-ray crystal structure of PS I from Synechocystis sp. PCC 6803 [2], the water molecule serving as an axial ligand for the $\mathrm{Chl}_{2 \mathrm{~A}}$ macrocycle is coordinated by two hydrogen bonds with the carbonyl oxygen atom of PsaB-N582 (H-acceptor) and with the indole nitrogen of PsaB-W586 (H-donor), which tightly arrange the $\mathrm{Chl}_{2 \mathrm{~A}}$ binding pocket (Fig. 9). However, the tryptophan residue PsaB-W586 has no analog in the $\mathrm{Chl}_{2 \mathrm{~B}}$ binding site, and the water molecule that serves as an axial ligand for the $\mathrm{Chl}_{2 \mathrm{~B}}$ macrocycle is coordinated only by one hydrogen bond with the carbonyl oxygen of PsaA-N600. This may be the reason why only two $\mathrm{Chl}_{2 \mathrm{~B}}$ macrocycles in the trimeric complex are ligated by water, while the $C h l_{2 \mathrm{~B}}$ molecule \#7131 has no axial ligand and the respective carbonyl oxygen of PsaA-N600 departs from the magnesium atom of the $C h l_{2 \mathrm{~B}}$ ring [2]. 
The substitution of Met for Asn in both PsaA-N600M and PsaB-N582M variants had a small effect on the rate of $A_{0} \rightarrow A_{1}$ electron transfer (Table 1, Fig. 6). However, in the PsaB-N582M variant, considerable kinetic heterogeneity was revealed by the CONTIN analysis, which indicated the presence of the kinetic component attributed to the excitation quenching in a small fraction of the PS I complexes (marked by the white box in Fig. 6f). The contribution of this component substantially increased in the PsaB-N582H and especially in the PsaB-N582L variant (Fig. 6 g, h), where the quantum yields of $\mathrm{P}_{700}{ }^{+} \mathrm{A}_{0}{ }^{-}$and $\mathrm{P}_{700}{ }^{+} \mathrm{A}_{1}{ }^{-}$formation were 0.64 and 0.4 , respectively (Table 2 ). The moderately decreased quantum yield of 0.64 was also found for the PsaA-N600L variant, but the CONTIN analysis did not reveal the expected kinetic component, which was hindered by the absorption recovery of the $\mathrm{A}_{0}$ band at $690 \mathrm{~nm}$ that proceeded in this variant at almost the same rate as in WT complexes (Table 2).

The strongest decrease in the quantum yield of the $\mathrm{P}_{700}{ }^{+} \mathrm{A}_{1}{ }^{-}$state was observed in the PsaB-N591L variant with the modified surroundings of $\mathrm{Chl}_{2 \mathrm{~A}}(\mathrm{Q} . \mathrm{Y} .=0.4)$; in this variant a significant distortion of the $\mathrm{P}_{700}{ }^{+} \mathrm{A}_{1}{ }^{-}$spectrum was observed (Fig. 5b, dark blue). Decreased quantum yields were found also in the PsaB-N582H (Q.Y.=0.64) and PsaAN600L (Q.Y. $=0.64)$ variants with the modified surroundings of $C h l_{2 A}$ and $C h l_{2 B}$, respectively, but the $\mathrm{P}_{700}{ }^{+} \mathrm{A}_{1}{ }^{-}$spectra of these complexes were relatively undistorted (Figs. $4 \mathrm{~b}$ and 5a). The quantum yields of charge separation in the PsaA-N604H/L and PsaB-N591H/L complexes were determined by Badshah et al. [29] using two complementary approaches. First, the quantum yield was determined by the extent of slow $\mathrm{P}_{700}{ }^{+}$reduction measured at $820 \mathrm{~nm}$ (the $\Delta \mathrm{A}_{820}$ column in Table 2). Second, the quantum yield was calculated by the $\mathrm{P}_{700}{ }^{+}$bleach amplitude measured at $703 \mathrm{~nm}$ in the final $\mathrm{P}_{700}{ }^{+} \mathrm{A}_{1}{ }^{-}$transient spectrum obtained by femtosecond spectroscopy (the $\Delta \mathrm{A}_{703}$ column in Table 2). While the quantum yields of $\mathrm{P}_{700}{ }^{+} \mathrm{A}_{1}{ }^{-}$formation calculated by a change of the Soret band bleach at $440 \mathrm{~nm}$ in this work are compatible with those of Badshah et al. [29] for some of the PS I variants, a significant slowing of the $\mathrm{A}_{0} \rightarrow \mathrm{A}_{1}$ transition in the PsaB-N582L and PsaB-N582H complexes compared to the WT allows us to draw different conclusions on the functioning of the second pair $\mathrm{Chl}_{2 A} / \mathrm{Chl}_{2 B}$ molecules in the course of electron transfer that ultimately branches into the two symmetric pathways.

\subsection{Nonphotochemical excitation quenching in photosystem I}

Quenching of Chl fluorescence by PS I complexes with oxidized $\mathrm{P}_{700}$ ("closed" centers) proceeds at almost the same rate as energy trapping by normal PS I complexes with neutral $\mathrm{P}_{700}$ ("open" centers) [53, 54]. Figure 8 shows that in the PsaB-N582L variant, where the nonproductive excitation quenching was maximal among the six PS I variants, a substantial absorption recovery was observed at the time range of $10 \mathrm{ps}$, both in the Soret region $(440 \mathrm{~nm})$ and the red edge of $\mathrm{Q}_{\mathrm{Y}}$ band $(710 \mathrm{~nm})$, where major absorption changes were observed also in the closed WT PS I complexes.

The strange coincidence of the quenching kinetics in the open and closed PS I complexes was rationalized by a model where the trapping kinetics in both cases are limited by excitation energy transfer from the LHA to the RC [62, 63]. The key role of the $\mathrm{P}_{700}{ }^{+}$cation as a major quencher for the excited $\mathrm{Chl}$ in the antenna was established by measuring the temperature dependence of the PS I fluorescence intensity both in the open and closed states [64]. Upon lowering the temperature to $77 \mathrm{~K}$, the fluorescence yield increased by a factor of 40 for PS I with neutral $\mathrm{P}_{700}$, and only by a factor of $\sim 4$ for PS I with oxidized $\mathrm{P}_{700}$. The high efficiency of excitation quenching by $\mathrm{P}_{700}{ }^{+}$was explained by a broad absorption band of the $\mathrm{P}_{700}$ cation in the farred region, which overlaps with the emission bands of the low-energy $\mathrm{Chl}$ of the antenna (Förster inductive resonance energy transfer) [64].

This transfer-to-trap-limited mechanism implies that the primary charge separation reactions in open PS I $\left(k_{1}\right)$ and the non-radiative energy dissipation by the excited $\left(\mathrm{P}_{700}{ }^{+}\right)^{*}$ in closed PS I $\left(k_{\mathrm{d}}\right)$ both are significantly faster than the excited energy transfer $\left(k_{\mathrm{EET}}\right)$ occurring at the time scale of few picoseconds [62]:

$$
\begin{aligned}
& L H A^{*} \stackrel{k_{\mathrm{EET}}}{\longrightarrow}\left(P_{700} A_{0}\right)^{*} \stackrel{k_{1}}{\longrightarrow} P_{700}^{+} A_{0}^{-} \stackrel{k_{2}}{\longrightarrow} P_{700}^{+} A_{1}^{-} \quad \text { (open) } \\
& L H A^{*} \stackrel{k_{\mathrm{EET}}}{\longrightarrow}\left(P_{700}^{+} A_{0}\right)^{*} \stackrel{k_{\mathrm{d}}}{\longrightarrow} P_{700}^{+} A_{0} \quad \text { (closed). }
\end{aligned}
$$

An ultrafast formation of the primary ion-radical pair $\mathrm{P}_{700}{ }^{+} \mathrm{A}_{0}{ }^{-}$was inferred from femtosecond absorption changes induced by PS I excitation in the far-red region [12, 13]. A fast non-radiative $S_{1} \rightarrow S_{0}$ conversion of excited positively charge porphyrin macrocycles in the picosecond timescale was observed in complexes with a strong out-of-plane distortion of the porphyrin structure [65-67]. The porphyrin molecules of the $\mathrm{P}_{700}$ dimer and the $\mathrm{Chl}_{2 \mathrm{~A}} / \mathrm{Chl}_{2 \mathrm{~B}}$ monomers are all extremely distorted out of the macrocycles planes (Fig. 9), which should provide strong electron-phonon coupling required for the fast non-radiative $\left(\mathrm{P}_{700}{ }^{+}\right)^{*} \rightarrow\left(\mathrm{P}_{700}{ }^{+}\right)$ conversion.

It should be noted that the unproductive energy dissipation observed in the PsaA-N600L, PsaB-N582H, and PsaB-N582L variants cannot occur by exactly the same mechanism as in closed PS I, since the special pair in these experiments was in the neutral state (open centers). Two possible scenarios can be considered.

In the first scenario, the primary ion-radical pair is normally formed in the altered PS I variants, but the secondary 
charge transfer reaction is hindered, $k_{2}^{\prime}<k_{2}$, and a partial charge recombination $\mathrm{P}_{700}{ }^{+} \mathrm{A}_{0}{ }^{-} \rightarrow \mathrm{P}_{700} \mathrm{~A}_{0}$ accounts for the decreased quantum yield in these variants:

$L H A^{*} \stackrel{k_{\mathrm{EET}}}{\longrightarrow}\left(P_{700} A_{0}\right)^{*} \stackrel{k_{1}}{\longrightarrow} P_{700}^{+} A_{0}^{-}\left\{\begin{array}{l}\stackrel{k_{2}^{\prime}}{\longrightarrow} P_{700}^{+} A_{1}^{-} \\ \stackrel{k_{r}}{\longrightarrow} P_{700} A_{0}\end{array}\right.$

In the second scenario, the amino acid substitution impedes formation of the primary $\mathrm{P}_{700}{ }^{+} \mathrm{A}_{0}{ }^{-}$ion-radical pair, $k_{1}^{\prime}<k_{1}$, and energy dissipation proceeds due to a nonradiative $S_{1} \rightarrow S_{0}$ conversion of the excited special pair:

$L H A^{*} \stackrel{k_{\mathrm{EET}}}{\longrightarrow}\left(P_{700} A_{0}\right)^{*}\left\{\begin{array}{l}\stackrel{k_{1}^{\prime}}{\longrightarrow} P_{700}^{+} A_{0}^{-} \stackrel{k_{2}}{\longrightarrow} P_{700}^{+} A_{1}^{-} . \\ \stackrel{k_{d}^{\prime}}{\longrightarrow} P_{700} A_{0}\end{array}\right.$

Analysis of the initial $S_{0}(\lambda)$ spectra registered in five PS I variants reviled a decreased yield of the ion-radical pair $\mathrm{P}_{700}{ }^{+} \mathrm{Chl}_{2}{ }^{-}$formation at the shortest delay of $100 \mathrm{fs}$ [35] The first scenario seems to be also less probable, because it implies a fast nonadiabatic recombination of the $\mathrm{P}_{700}{ }^{+} \mathrm{A}_{0}{ }^{-}$ion-radical pair, which normally occurs on the time scale of $20 \mathrm{~ns}$ [68]. Because the forward $\left(\mathrm{P}_{700} \mathrm{~A}_{0}\right) * \rightarrow \mathrm{P}_{700}{ }^{+} \mathrm{A}_{0}{ }^{-}$transition is extremely fast $[12,13]$ being driven by a small energy gap of $\sim 40 \mathrm{meV}$ [14], the reorganization energy of $\mathrm{P}_{700}{ }^{+} \mathrm{A}_{0}{ }^{-}$formation is also small. On the contrary, the energy gap for backward $\mathrm{P}_{700}{ }^{+} \mathrm{A}_{0}{ }^{-} \rightarrow \mathrm{P}_{700} \mathrm{~A}_{0}$ recombination is about $1.6 \mathrm{eV}$, so the backward reaction is far in the inverted region, which inevitably will slow down the recombination [69].

The dimers of perylene are often considered as chemical models for charge separation reactions in photosynthetic reaction centers. Electron transfer in the isomeric $\mathrm{N}$-annulated perylene diimide dimer (paraBDNP) may be considered as a suitable prototype for $\left(\mathrm{P}_{700} \mathrm{~A}_{0}\right)^{*} \rightarrow \mathrm{P}_{700}{ }^{+} \mathrm{A}_{0}{ }^{-} \rightarrow \mathrm{P}_{700} \mathrm{~A}_{0}$ transitions in PS I: the center-to-center distance between monomers in paraBDNP is $11.5 \AA$, the driving force for the charge separation in slightly polar tetrahydrofuran is $-0.2 \mathrm{eV}$, the forward charge separation and backward recombination lifetimes were determined for this complex as $12 \mathrm{ps}$ and $1.9 \mathrm{~ns}$, respectively [70]. Similar lifetimes of 1-2 ns were obtained for recombination of $\mathrm{Chl}^{-}-\mathrm{Chl}^{+}$ion pairs formed in the light-harvesting complex LHCII [71]. All these examples of nonadiabatic recombination are two-three orders of magnitude slower than the lifetime of $10 \mathrm{ps}$ determined for energy dissipation in the PsaA-N600L, PsaB-N582H, and PsaB-N582L variants (Fig. 6).

The second scenario (Eq. 10) is analogous to the mechanism of excitation quenching in closed PS I, but the nonradiative $S_{1} \rightarrow S_{0}$ conversion is driven here by the neutral state of the excited special pair. This scenario also has certain difficulties, since the typical lifetime for non-radiative $\mathrm{S}_{1} \rightarrow \mathrm{S}_{0}$ conversion of monomeric Chl $a$ is $6 \mathrm{~ns}$ [72].

The electronic structure of $\mathrm{Chl} a$ is conventionally approximated by the four-orbital Gouterman model, where the two electronic transitions $\mathrm{Q}_{\mathrm{Y}}\left(\right.$ or $\left.\mathrm{S}_{1}\right)$ and $\mathrm{Q}_{\mathrm{X}}\left(\right.$ or $\left.\mathrm{S}_{2}\right)$ from the highest occupied (HOMO) and the next below (HOMO1) molecular orbitals to the lowest unoccupied molecular orbital (LUMO) comprise the low-energy Q band, and the analogous transitions $\mathrm{B}_{\mathrm{X}}$ (or $\mathrm{S}_{3}$ ) and $\mathrm{B}_{\mathrm{Y}}\left(\right.$ or $\mathrm{S}_{4}$ ) to the next unoccupied molecular orbital $(\mathrm{LUMO}+1)$ comprise the high-energy B band (also named as the Soret band) [73]. The relaxation of high-energy states $S_{3}$ and $S_{2}$ to the lowest excited state $S_{1}$ takes place on the time scale of 100-300 fs [46], but the non-radiative conversion to the ground state $\mathrm{S}_{1} \rightarrow \mathrm{S}_{0}$ occurs in different solutions at 5-7 ns [72].

An effective conversion of the $\mathrm{S}_{1}$ excitation energy $h \nu \approx$ $1.6 \mathrm{eV}$ to the vibrational motion of $\mathrm{Chl}$ nucleus on the time scale of 10 ps requires strong electron-phonon coupling [74, $75]$, namely, the nuclear reorganization energy $(\lambda)$ of the $\mathrm{S}_{1} \rightarrow \mathrm{S}_{0}$ transition should be comparable with $1.6 \mathrm{eV}$. The reorganization energies of nonplanar porphyrins increase linearly relative to out-of-plane macrocycle displacements, reaching the maximal value for saddle-distorted dodecaphenylporphyrin [76]. The effects of macrocycle deformations on the rate of $S_{1} \rightarrow S_{0}$ conversion of substituted nonplanar porphyrins have been extensively studied $[65,77$, 78]. Whereas the $S_{1}$ lifetimes of the planar porphyrins are 10-15 ns, nonplanar deformations progressively increased both the Stokes shifts and the rates of the non-radiative conversion: in the ruffled porphyrins ultrashort $S_{1}$ lifetimes of 10-50 ps were measured at $296 \mathrm{~K}$ [79]. The strongly outof-plane distorted porphyrin macrocycles of tightly conjugated $\mathrm{P}_{700}, C h l_{2 \mathrm{~A}}$ and $C h l_{2 \mathrm{~B}}$ (Fig. 9) can function as effective catalytic centers for such non-radiative $S_{1} \rightarrow S_{0}$ conversions.

\subsection{Models of electron redistribution between symmetrical branches}

Due to the high $\mathrm{C}_{2}$-symmetry of the PS I heterodimer, both electron transfer chains demonstrate comparable functional activity [16, 18-28]. Two basic models of the chain branching mechanism have been suggested: a "donor-side equilibrium model" and a "branch competition model" [19]. In the first model, the direction of electron transfer along either $A$ or $B$ branch is determined by the two alternative static PS I conformations. In this model, the functioning of the branches is independent of each other, so the lockout of electron transfer along one of the branches due to an amino acid substitution leads to a total decrease in the quantum yield. In the second model, both branches compete for the electron sheared between the six redox-active cofactors; therefore, a decrease in the efficiency of electron transfer along one of 
the branches is compensated by the increased contribution of the symmetric branch, and the total quantum yield does not decrease.

The results obtained with different altered PS I variants provide alternating support for both mechanisms. The substitution PsaA-Y696F, which breaks the H-bond to $\mathrm{Chl}_{3 \mathrm{~A}}$, suppressed the formation of the $\mathrm{P}_{700}{ }^{+} \mathrm{Chl}_{3 \mathrm{~A}}{ }^{-}$, but this effect was compensated by an enhanced electron transfer along the $B$-branch; the reciprocal substitution PsaB-Y676F suppressed electron transfer along the $B$-branch and increased the contribution of the $A$-branch [19]. These data are in agreement with the observation of the fast electron redistribution between the two branches in PS I complexes from C. reinhardtii $[14,16]$ and the cyanobacteria Thermosynechococcus elongatus and Synechocystis sp. PCC 6803 [44]. On the other hand, the decreased quantum yields of charge separation in PS I complexes from C. reinhardtii and Synechocystis sp. PCC 6803 with the PsaB-N591L and PsaAN604L substitutions (amino acid numbering according to the sequence of $C$. reinhardtii) were interpreted in favor of the "donor-side equilibrium model" [29]. The latter conclusion was based on the experimental result that the $\mathrm{A}_{0} \rightarrow \mathrm{A}_{1}$ electron transfer in the PsaB-N591L and PsaAN604L variants occurred with the decay time of 21-35 ps, i.e., similar to the wild type. Hence, the decreased quantum yield for these variants was explained as complete blocking of charge separation in a certain fraction of the PS I complexes, whereas in the other fraction the electron transfer occurred via the unaffected branch [29].

The critical analysis of the absorption changes of the PsaB-N582H and PsaB-N582L variants performed in this work demonstrates that the $\mathrm{A}_{0} \rightarrow \mathrm{A}_{1}$ transition is slowed about threefold compared to the WT, while in the PsaAN600M/H/L and PsaB-N582M variants the slowing was only by a factor of 1.3 (Table 1 ). In the PsaB-N582H and PsaB-N582L variants the deceleration was accompanied by a significant decrease in the quantum yield of the secondary $\mathrm{P}_{700}{ }^{+} \mathrm{A}_{1}{ }^{-}$ion-radical pair the magnitude of which is consistent with the data by Badshah et al. [29] (Table 2). In addition, an alternative pathway of the excited state trapping with the decay time of 8-25 ps was inferred from the absorption dynamics at $\sim 710 \mathrm{~nm}$ attributed to the antenna chlorophyll (Fig. 8). The similar excitation trapping on the time scale of 7-25 ps has been observed in the "closed" PS I complexes with the pre-oxidized $\mathrm{P}_{700}$ $[10,14,53]$.

As follows from Figs. $5 \mathrm{~b}, 6 \mathrm{~h}$, the $\mathrm{A}_{0} \rightarrow \mathrm{A}_{1}$ transition in the PsaB-N582L variant (measured as recovery of the sharp bleach at $690 \mathrm{~nm}$ ) has a decay time of $\sim 70$ ps. Because the quantum yield of the $\mathrm{P}_{700}{ }^{+} \mathrm{A}_{1}{ }^{-}$state in this variant is $\sim 40 \%$, the charge separation proceeds predominantly via the $A$-branch with the altered $C h l_{2 A}$, and it means that electron redistribution between the two branches in favor of the $A$-branch takes place in a ten picosecond time scale as previously suggested $[16,19,44]$. Thus, the data as a whole support the "branch competition model".

The symmetry breaking in this model is a result of the electron density localization in one of the $A$ - and $B$-branches obliged by protein polarization. This mechanism is in agreement with an adiabatic model, where the $\mathrm{Chl}$ dimer $\mathrm{P}_{700}$ is electronically coupled with the symmetrically arranged monomers $C h l_{2 \mathrm{~A}}$ and $C h l_{2 \mathrm{~B}}[13,80]$. Such a structure can be considered as a symmetric exciplex $C h l_{2 \mathrm{~A}} P_{\mathrm{A}} P_{\mathrm{B}} C h l_{2 \mathrm{~B}}$ in which the excited state $\left(\mathrm{Chl}_{2 \mathrm{~A}} P_{\mathrm{A}} P_{\mathrm{B}} C h l_{2 \mathrm{~B}}\right)^{*}$ is mixed with two charge-transfer states $P_{700}{ }^{+} \mathrm{Chl}_{2 \mathrm{~A}}{ }^{-}$and $P_{700}{ }^{+} \mathrm{Chl}_{2 \mathrm{~B}}{ }^{-}$[35]. In the framework of the symmetric exciplex model, the redistribution of electron between the two $\mathrm{Chl}$ branches is expected before the irreversible step of phylloquinone reduction. The functional asymmetry in favor of the A-branch in the PS I structure from Synechocystis sp. PCC 6803 [2] may arise due to differences in the hydrogen bond networks near the $C h l_{2 \mathrm{~A}}$ and $C h l_{2 \mathrm{~B}}$ monomers: the formation of $C h l_{2 \mathrm{~A}}$ anion is stabilized by polar tryptophan PsaB-W586, which serves as an $\mathrm{H}$-donor for the axial ligand of $\mathrm{Chl}_{2 \mathrm{~A}}$; the homologous hydrophobic isoleucine PsaA-I604 does not form H-bonds in the $C h l_{2 \mathrm{~B}}$ binding cavity, which should impede the reduction of $C h l_{2 \mathrm{~B}}$.

The substitutions PsaA-N600M/H/L and PsaBN582M hinder the formation of the charge-transfer state $P_{700}{ }^{+} \mathrm{Chl}_{2 \mathrm{~B}}{ }^{-}$or $\mathrm{P}_{700}{ }^{+} \mathrm{Chl}_{2 \mathrm{~A}}{ }^{-}$in the altered branch and increase the effective time of the $\mathrm{A}_{0} \rightarrow \mathrm{A}_{1}$ transition by a factor of 1.3 from $24 \mathrm{ps}$ in the WT to $30-34 \mathrm{ps}$. A decrease in the total quantum yield of charge separation in the PsaB$\mathrm{N} 582 \mathrm{H}$ and PsaB-N582L variants correlates with the threefold slowing of the phylloquinone $\mathrm{A}_{0} \rightarrow \mathrm{A}_{1}$ reduction, but not with the absorption changes at the shortest delay assigned to the $\mathrm{P}_{700}{ }^{+} \mathrm{Chl}_{2 \mathrm{~A}}{ }^{-}$and $\mathrm{P}_{700}{ }^{+} \mathrm{Chl}_{2 \mathrm{~B}}{ }^{-}$charge-transfer states. The decreased quantum yield of charge separation in these variants may, therefore, be due to the process of radiationless $\mathrm{S}_{1} \rightarrow \mathrm{S}_{0}$ conversion within the out-of-plane distorted $\mathrm{P}_{700}$, $\mathrm{Chl}_{2 \mathrm{~A}}$ and $\mathrm{Chl}_{2 \mathrm{~B}}$ macrocycles or through the nonadiabatic recombination of $\mathrm{Chl}^{-}-\mathrm{Chl}^{+}$ion-radical pairs in the antenna chlorophyll.

\section{Conclusions}

The femtosecond absorption changes of PS I complexes carrying three pairs of complementary amino acid substitutions near the second pair of Chl molecules (Asn $\rightarrow$ Met, His, Leu) were analyzed by decompositions of the transition spectra into discrete decay-associated spectra and continuously distributed exponential components. The multi-exponential deconvolution of the absorption dynamics revealed that the electron transfer reactions in the PsaA-N600M, PsaA$\mathrm{N} 600 \mathrm{H}$, and PsaA-N600L variants with the altered $B$-branch 
of cofactors are generally similar to those of the wild type, while the PsaB-N582M, PsaB-N582H, and PsaB-N582L substitutions, which alter the $A$-branch of cofactors, cause significant alterations of the photochemical processes, making them heterogeneous and poorly described by a discrete exponential kinetic model. A redistribution of unpaired electron between the $\mathrm{Chl}_{2 \mathrm{~A}} / \mathrm{Ch} l_{2 \mathrm{~B}}$ and $\mathrm{Chl}_{3 \mathrm{~A}} / \mathrm{Chl}_{3 \mathrm{~B}}$ molecules limited by excitation energy transfer from the antenna to the $\mathrm{RC}$ was identified in the time range of 9-20 ps, and the subsequent reduction of $A_{1}$ in the time range of 24-70 ps. In the PsaA-N600L and PsaB-N582H/L variants, the reduction of $A_{1}$ occurred with a decreased quantum yield. In the absorption dynamics of the PsaB-N582H and PsaBN582L variants, a specific kinetic component $(\tau=10-30 \mathrm{ps,}$ $\lambda=700-720 \mathrm{~nm}$ ) was identified as associated with excitation quenching in the light-harvesting antenna. The decreased quantum yield of charge separation in the PsaA-N600L, PsaB-N582H, and PsaB-N582L variants does not correlate with the initial transient spectra measured at the shortest time delay. In general, the results can be interpreted in the framework of "branch competition model", where the electron is shared between the six redox-active Chl cofactors before its transfer to phylloquinone in either $\mathrm{A}_{1 \mathrm{~A}}$ or $\mathrm{A}_{1 \mathrm{~B}}$ sites.

Supplementary Information The online version contains supplementary material available at https://doi.org/10.1007/s43630-021-00094-y.

Acknowledgements This work was supported by the Russian Science Foundation Grant RSF 19-14-00366 to DAC and AYS and by the US National Science Foundation under grant MCB-1613022 to JHG. Cell growth and isolation of the PS I complexes was funded by NSF EPSCoR (NSF(2010)-PFUND-217) and the Louisiana RCS Program (LEQSF(2013-16)-RD-A-15) to WX. AYS acknowledges partial support from Lomonosov Moscow State University Program of Development. We thank Dr. Vasily Kurashov and Dr. Michael Gorka for valuable discussions.

\section{Declarations}

Conflict of interest The authors have no conflicts of interest to declare.

\section{References}

1. Jordan, P., Fromme, P., Witt, H. T., Klukas, O., Saenger, W., \& Krauß, N. (2001). Three-dimensional structure of cyanobaoterial photosystem I at 2.5 A resolution. Nature, 411, 909-917.

2. Mazor, Y., Nataf, D., Toporik, H., \& Nelson, N. (2014). Crystal structures of virus-like photosystem I complexes from the mesophilic cyanobacterium Synechocystis PCC 6803. eLife, 3, e01496.

3. Mazor, Y., Borovikova, A., \& Nelson, N. (2017). The structure of plant photosystem I super-complex at 2.8 A resolution. Nature Plants, 3, 17014.

4. Gorka, M., Charles, P., Kalendra, V., Baldansuren, A., Lakshmi, K. V., \& Golbeck, J. H. (2021). A dimeric chlorophyll electron acceptor differentiates type I from type II photosynthetic reaction centers. iScience, 24, 102719.

5. Melkozernov, A. N., Lin, S., \& Blankenship, R. E. (2000). Excitation dynamics and heterogeneity of energy equilibration in the core antenna of photosystem I from the Cyanobacterium Synechocystis sp. PCC 6803. Biochemistry, 39, 1489-1498.

6. Savikhin, S., \& Jankowiak, R. (2014). In J. Golbeck \& A. van der Est (Eds.), The biophysics of photosynthesis (pp. 193-240). Springer.

7. Di Donato, M., Stahl, A. D., Van Stokkum, I. H. M., Van Grondelle, R., \& Groot, M. L. (2011). Cofactors involved in lightdriven charge separation in photosystem I identified by subpicosecond infrared spectroscopy. Biochemistry, 50, 480-490.

8. Müller, M. G., Niklas, J., Lubitz, W., \& Holzwarth, A. R. (2003). Ultrafast transient absorption studies on photosystem I reaction centers from Chlamydomonas reinhardtii. 1. A new interpretation of the energy trapping and early electron transfer steps in photosystem I. Biophysical Journal, 85, 3899-3922.

9. Nürnberg, D. J., Morton, J., Santabarbara, S., Telfer, A., Joliot, P., Antonaru, L. A., Ruban, A. V., Cardona, T., Krausz, E., Boussac, A., Fantuzzi, A., \& Rutherford, A. W. (2018). Photochemistry beyond the red limit in chlorophyll $\mathrm{f}-$ containing photosystems. Science, 360, 1210-1213.

10. Russo, M., Petropoulos, V., Molotokaite, E., Cerullo, G., Casazza, A. P., Maiuri, M., \& Santabarbara, S. (2020). Ultrafast excited-state dynamics in land plants Photosystem I core and whole supercomplex under oxidised electron donor conditions. Photosynthesis Research, 144, 221-233.

11. Gibasiewicz, K., Ramesh, V. M., Melkozernov, A. N., Lin, S., Woodbury, N. W., Blankenship, R. E., \& Webber, A. N. (2001). Excitation dynamics in the core antenna of PS I from Chlamydomonas reinhardtii CC 2696 at room temperature. The Journal of Physical Chemistry B, 105, 11498-11506.

12. Shelaev, I. V., Gostev, F. E., Mamedov, M. D., Sarkisov, O. M., Nadtochenko, V. A., Shuvalov, V. A., \& Semenov, A. Y. (2010). Femtosecond primary charge separation in Synechocystis sp. PCC 6803 photosystem I. Biochimica et Biophysica Acta Bioenergetics, 1797, 1410-1420.

13. Cherepanov, D. A., Shelaev, I. V., Gostev, F. E., Mamedov, M. D., Petrova, A. A., Aybush, A. V., Shuvalov, V. A., Semenov, A. Y., \& Nadtochenko, V. A. (2017). Mechanism of adiabatic primary electron transfer in photosystem I: Femtosecond spectroscopy upon excitation of reaction center in the far-red edge of the Q Y band. Biochimica et Biophysica Acta Bioenergetics, $1858,895-905$.

14. Giera, W., Ramesh, V. M., Webber, A. N., van Stokkum, I., van Grondelle, R., \& Gibasiewicz, K. (2010). Effect of the P700 preoxidation and point mutations near A0 on the reversibility of the primary charge separation in Photosystem I from Chlamydomonas reinhardtii. Biochimica et Biophysica Acta Bioenergetics, 1797, 106-112.

15. Molotokaite, E., Remelli, W., Casazza, A. P., Zucchelli, G., Polli, D., Cerullo, G., \& Santabarbara, S. (2017). Trapping dynamics in Photosystem I-light harvesting complex I of higher plants is governed by the competition between excited state diffusion from low energy states and photochemical charge separation. The Journal of Physical Chemistry B, 121, 9816-9830.

16. Müller, M. G., Slavov, C., Luthra, R., Redding, K. E., \& Holzwarth, A. R. (2010). Independent initiation of primary electron transfer in the two branches of the photosystem I reaction center. Proceedings of the National Academy of Sciences of the United States of America, 107, 4123-4128.

17. Zhu, J., Van Stokkum, I. H. M., Paparelli, L., Jones, M. R., \& Groot, M. L. (2013). Early bacteriopheophytin reduction in charge separation in reaction centers of Rhodobacter sphaeroides. Biophysical Journal, 104, 2493-2502. 
18. Guergova-Kuras, M., Boudreaux, B., Joliot, A., Joliot, P., \& Redding, K. (2001). Evidence for two active branches for electron transfer in photosystem I. Proceedings of the National Academy of Sciences of the United States of America, 98, 4437-4442.

19. Li, Y., Van Der Est, A., Lucas, M. G., Ramesh, V. M., Gu, F., Petrenko, A., Lin, S., Webber, A. N., Rappaport, F., \& Redding, K. (2006). Directing electron transfer within Photosystem I by breaking $\mathrm{H}$-bonds in the cofactor branches. Proceedings of the National Academy of Sciences of the United States of America, 103, 2144-2149.

20. Gibasiewicz, K., Ramesh, V. M., Lin, S., Redding, K., Woodbury, N. W., \& Webber, A. N. (2003). Excitonic interactions in wildtype and mutant PSI reaction centers. Biophysics Journal, 85, 2547-2559.

21. Ramesh, V. M., Gibasiewicz, K., Lin, S., Bingham, S. E., \& Webber, A. N. (2004). Bidirectional electron transfer in photosystem I: accumulation of A0- in A-side or B-side mutants of the axial ligand to chlorophyll A0. Biochemistry, 43, 1369-1375.

22. Santabarbara, S., Kuprov, I., Fairclough, W. V., Purton, S., Hore, P. J., Heathcote, P., \& Evans, M. C. W. (2005). Bidirectional electron transfer in photosystem I: Determination of two distances between $\mathrm{P} 700+$ and A1- in spin-correlated radical pairs. Biochemistry, 44, 2119-2128.

23. Giera, W., Gibasiewicz, K., Ramesh, V. M., Lin, S., \& Webber, A. (2009). Electron transfer from A0 to A1 in Photosystem i from Chlamydomonas reinhardtii occurs in both the A and B branch with 25-30-ps lifetime. Physical Chemistry Chemical Physics: PCCP, 11, 5186-5191.

24. Ramesh, V. M., Gibasiewicz, K., Lin, S., Bingham, S. E., \& Webber, A. N. (2007). Replacement of the methionine axial ligand to the primary electron acceptor A0 slows the A0- reoxidation dynamics in Photosystem I. Biochimica et Biophysica Acta Bioenergetics, 1767, 151-160.

25. Dashdorj, N., Xu, W., Cohen, R. O., Golbeck, J. H., \& Savikhin, S. (2005). Asymmetric electron transfer in cyanobacterial photosystem I: Charge separation and secondary electron transfer dynamics of mutations near the primary electron acceptor A0. Biophysical Journal, 88, 1238-1249.

26. Cohen, R. O., Shen, G., Golbeck, J. H., Xu, W., Chitnis, P. R., Valieva, A. I., Van Der Est, A., Pushkar, Y., \& Stehlik, D. (2004). Evidence for asymmetric electron transfer in cyanobacterial photosystem I: Analysis of a methionine-to-leucine mutation of the ligand to the primary electron acceptor A0. Biochemistry, 43, $4741-4754$.

27. Santabarbara, S., Kuprov, I., Poluektov, O., Casal, A., Russell, C. A., Purton, S., \& Evans, M. C. W. (2010). Directionality of electron-transfer reactions in photosystem I of prokaryotes: Universality of the bidirectional electron-transfer model. The Journal of Physical Chemistry B, 114, 15158-15171.

28. Sun, J., Hao, S., Radle, M., Xu, W., Shelaev, I., Nadtochenko, V., Shuvalov, V., Semenov, A., Gordon, H., van der Est, A., \& Golbeck, J. H. (2014). Evidence that histidine forms a coordination bond to the $\mathrm{A} 0 \mathrm{~A}$ and $\mathrm{A} 0 \mathrm{~B}$ chlorophylls and a second $\mathrm{H}$-bond to the A1A and A1B phylloquinones in M688HPsaA and M668HPsaB variants of Synechocystis sp PCC 6803. Biochimica et Biophysica Acta Bioenergetics, 1837, 1362-1375.

29. Badshah, S. L., Sun, J., Mula, S., Gorka, M., Baker, P., Luthra, R., Lin, S., van der Est, A., Golbeck, J. H., \& Redding, K. E. (2018). Mutations in algal and cyanobacterial Photosystem I that independently affect the yield of initial charge separation in the two electron transfer cofactor branches. Biochimica et Biophysica Acta Bioenergetics, 1859, 42-55.

30. Kurashov, V., Gorka, M., Milanovsky, G. E., Johnson, T. W., Cherepanov, D. A., Semenov, A. Y., \& Golbeck, J. H. (2018). Critical evaluation of electron transfer kinetics in P700-FA/FB, P700-FX, and P700-A1 Photosystem I core complexes in liquid and in trehalose glass. Biochimica et Biophysica Acta Bioenergetics, 1859, 1288-1301.

31. Srinivasan, N., \& Golbeck, J. H. (2009). Protein-cofactor interactions in bioenergetic complexes: the role of the A1A and A1B phylloquinones in Photosystem I. Biochimica Biophysica Acta, 1787, 1057-1088.

32. Cherepanov, D. A., Milanovsky, G. E., Petrova, A. A., Tikhonov, A. N., \& Semenov, A. Y. (2017). Electron transfer through the acceptor side of photosystem I: Interaction with exogenous acceptors and molecular oxygen. Biochemistry, 82, 1249-1268.

33. Brettel, K., \& Golbeck, J. H. (1995). Spectral and kinetic characterization of electron acceptor A1 in a photosystem I core devoid of iron-sulfur centers FX, FB and FA. Photosynthesis Research, 45, 183-193.

34. Makita, H., \& Hastings, G. (2015). Directionality of electron transfer in cyanobacterial photosystem I at 298 and 77 K. FEBS Letters, 589, 1412-1417.

35. Cherepanov, D. A., Shelaev, I. V., Gostev, F. E., Petrova, A., Aybush, A. V., Nadtochenko, V. A., Xu, W., Golbeck, J. H., \& Semenov, A. Y. (2021). Primary charge separation within the structurally symmetric tetrameric Chl2APAPBChl2B chlorophyll exciplex in photosystem I. Journal of Photochemistry and Photobiology B Biology, 217, 112154.

36. Xu, W., Chitnis, P., Valieva, A., Van der Est, A., Pushkar, Y. N., Krzystyniak, M., Teutloff, C., Zech, S. G., Bittl, R., Stehlik, D., Zybailov, B., Shen, G., \& Golbeck, J. H. (2003). Electron transfer in cyanobacterial photosystem I. I. Physiological and spectroscopic characterization of site-directed mutants in a putative electron transfer pathway from A0 through A1 to Fx. Journal of Biological Chemistry, 278, 27864-27875.

37. Dobryakov, A. L., Pérez Lustres, J. L., Kovalenko, S. A., \& Ernsting, N. P. (2008). Femtosecond transient absorption with chirped pump and supercontinuum probe: Perturbative calculation of transient spectra with general lineshape functions, and simplifications. Chemical Physics, 347, 127-138.

38. Golubeva, E. N., Zubanova, E. M., Melnikov, M. Y., Gostev, F. E., Shelaev, I. V., \& Nadtochenko, V. A. (2014). Femtosecond spectroscopy and TD-DFT calculations of $\mathrm{CuCl} 42-$ excited states. Dalton Transaction, 43, 17820-17827.

39. Provencher, S. W. (1982). CONTIN: A general purpose constrained regularization program for inverting noisy linear algebraic and integral equations. Computer Physics Communications, 27, 229-242.

40. Heimdal, J., Jensen, K. P., Devarajan, A., \& Ryde, U. (2007). The role of axial ligands for the structure and function of chlorophylls. Journal of Biological Inorganic Chemistry, 12, 49-61.

41. Rätsep, M., Johnson, T. W., Chitnis, P. R., \& Small, G. J. (2000). The red-absorbing chlorophyll a antenna states of photosystem I: A hole-burning study of Synechocystis sp. PCC 6803 and its mutants. Journal of Physical Chemistry B, 104, 836-847.

42. Herascu, N., Hunter, M. S., Shafiei, G., Najafi, M., Johnson, T. W., Fromme, P., \& Zazubovich, V. (2016). Spectral hole burning in cyanobacterial photosystem I with P700 in oxidized and neutral states. The Journal of Physical Chemistry B, 120, 10483-10495.

43. Witt, H., Bordignon, E., Carbonera, D., Dekker, J. P., Karapetyan, N., Teutloff, C., Webber, A., Lubitz, W., \& Schlodder, E. (2003). Species-specific differences of the spectroscopic properties of P700: analysis of the influence of non-conserved amino acid residues by site-directed mutagenesis of photosystem I from Chlamydomonas reinhardtii. Journal of Biological Chemistry, 278, 46760-46771.

44. Cherepanov, D. A., Shelaev, I. V., Gostev, F. E., Aybush, A. V., Mamedov, M. D., Shuvalov, V. A., Semenov, A. Y., \& Nadtochenko, V. A. (2020). Generation of ion-radical chlorophyll states in the light-harvesting antenna and the reaction center of 
cyanobacterial photosystem I. Photosynthetic Research, 146, $1-19$.

45. Cherepanov, D. A., Gostev, F. E., Shelaev, I. V., Aibush, A. V., Mamedov, M. D., Shuvalov, V. A., Semenov, A. Y., \& Nadtochenko, V. A. (2020). Visible and near infrared absorption spectrum of the excited singlet state of chlorophyll a. High Energy Chemistry, 54, 145-147.

46. Bricker, W. P., Shenai, P. M., Ghosh, A., Liu, Z., Enriquez, M. G. M., Lambrev, P. H., Tan, H. S., Lo, C. S., Tretiak, S., Fernandez-Alberti, S., \& Zhao, Y. (2015). Non-radiative relaxation of photoexcited chlorophylls: Theoretical and experimental study. Scientific Reports, 5, 1-16.

47. Lee, Y., Gorka, M., Golbeck, J. H., \& Anna, J. M. (2018). Ultrafast energy transfer involving the red chlorophylls of cyanobacterial photosystem I probed through two-dimensional electronic spectroscopy. Journal of the American Chemical Society, 140, 11631-11638.

48. Wasielewski, M. R., Fenton, J. M., \& Govindjee. (1987). The rate of formation of P700+ A0 in photosystem I particles from spinach as measured by picosecond transient absorption spectroscopy. Photosynthetic Research, 12, 181-189.

49. Mathis, P., Ikegami, I., \& Setif, P. (1988). Nanosecond flash studies of the absorption spectrum of the photosystem I primary acceptor Ao. Photosynthesis Research, 16, 203-210.

50. Shuvalov, V. A., Nuijs, A. M., van Gorkom, H. J., Smit, H. W. J., \& Duysens, L. N. M. (1986). Picosecond absorbance changes upon selective excitation of the primary electron donor P-700 in photosystem I. Biochimica et Biophysica Acta Bioenergetics, 850, 319-323.

51. Dashdorj, N., Xu, W., Martinsson, P., Chitnis, P. R., \& Savikhin, S. (2004). Electrochromic shift of chlorophyll absorption in photosystem I from Synechocystis sp. PCC 6803: a probe of optical and dielectric properties around the secondary electron acceptor. Biophysical Journal, 86, 3121-3130.

52. Hastings, G., Kleinherenbrink, F. A. M. M., Lin, S., McHugh, T. J., \& Blankenship, R. E. (1994). Observation of the reduction and reoxidation of the primary electron acceptor in photosystem I. Biochemistry, 33, 3193-3200.

53. Savikhin, S., Xu, W., Chitnis, P. R., \& Struve, W. S. (2000). Ultrafast primary processes in PS I from Synechocystis sp. PCC 6803: Roles of P700 and A0. Biophysical Journal, 79, 1573-1586.

54. Byrdin, M., Rimke, I., Schlodder, E., Stehlik, D., \& Roelofs, T. A. (2000). Decay kinetics and quantum yields of fluorescence in photosystem I from Synechococcus elongatus with P700 in the reduced and oxidized state: Are the kinetics of excited state decay trap-limited or transfer-limited? Biophysical Journal, 79, 992-1007.

55. Mi, D., Lin, S., \& Blankenship, R. E. (1999). Picosecond transient absorption spectroscopy in the blue spectral region of photosystem I. Biochemistry, 38, 15231-15237.

56. Brettel, K., \& Vos, M. H. (1999). Spectroscopic resolution of the picosecond reduction kinetics of the secondary electron acceptor A1 in photosystem I. FEBS Letters, 447, 315-317.

57. Cherepanov, D. A., Brady, N. G., Shelaev, I. V., Nguyen, J., Gostev, F. E., Mamedov, M. D., Nadtochenko, V. A., \& Bruce, B. D. (2020). PSI-SMALP, a detergent-free cyanobacterial photosystem I, reveals faster femtosecond photochemistry. Biophysical Journal, 118, 337-351.

58. Chauvet, A., Dashdorj, N., Golbeck, J. H., Johnson, T. W., \& Savikhin, S. (2012). Spectral resolution of the primary electron acceptor A0 in Photosystem I. Journal of Physical Chemistry B, 116, 3380-3386.

59. Kim, T., Kim, W., Mori, H., Osuka, A., \& Kim, D. (2018). Solvent and structural fluctuations induced symmetry-breaking charge transfer in a porphyrin triad. Journal of Physical Chemistry C, 122, 19409-19415.
60. Byrdin, M., Santabarbara, S., Gu, F., Fairclough, W. V., Heathcote, P., Redding, K., \& Rappaport, F. (2006). Assignment of a kinetic component to electron transfer between iron-sulfur clusters FX and FA/B of Photosystem I. Biochimica et Biophysica Acta Bioenergetics, 1757, 1529-1538.

61. Santabarbara, S., Jasaitis, A., Byrdin, M., Gu, F., Rappaport, F., \& Redding, K. (2008). Additive effect of mutations affecting the rate of phylloquinone reoxidation and directionality of electron transfer within photosystem I. Photochem. Photobiol., 84, 1381-1387.

62. Trissl, H. W. (1997). Determination of the quenching efficiency of the oxidized primary donor of Photosystem I, P700+: Implications for the trapping mechanism. Photosynthesis Research, 54, 237-240.

63. Brettel, K. (1997). Electron transfer and arrangement of the redox cofactors in photosystem I. Biochimica et Biophysica Acta Bioenergetics, 1318, 322-373.

64. Schlodder, E., Çetin, M., Byrdin, M., Terekhova, I. V., \& Karapetyan, N. V. (2005). P700 +- and 3P700-induced quenching of the fluorescence at $760 \mathrm{~nm}$ in trimeric Photosystem I complexes from the cyanobacterium Arthrospira platensis. Biochimica et Biophysica Acta Bioenergetics, 1706, 53-67.

65. Lebedev, A. Y., Filatov, M. A., Cheprakov, A. V., \& Vinogradov, S. A. (2008). Effects of structural deformations on optical properties of tetrabenzoporphyrins: Free-bases and Pd complexes. Journal of Physical Chemistry A, 112, 7723-7733.

66. Chang, M. H., Hoffmann, M., Anderson, H. L., \& Herz, L. M. (2008). Dynamics of excited-state conformational relaxation and electronic delocalization in conjugated porphyrin oligomers. Journal of the American Chemical Society, 130, 10171-10178.

67. Gentemann, S., Medforth, C. J., Forsyth, T. P., Nurco, D. J., Smith, K. M., Fajer, J., \& Holten, D. (1994). Photophysical properties of conformationally distorted metal-free porphyrins investigation into the deactivation mechanisms of the lowest excited singlet state. Journal of American Chemical Society, 116, 7363-7368.

68. Brettel, K., \& Sétif, P. (1987). Magnetic-field effects on primary reactions in Photosystem I. Biochimica Biophysica Acta - Bioenergetics, 893, 109-114.

69. Makita, H., \& Hastings, G. (2017). Inverted-region electron transfer as a mechanism for enhancing photosynthetic solar energy conversion efficiency. Proceedings of the National Academy of Sciences of the United States of America, 114, 9267-9272.

70. Guo, Y., Ma, Z., Niu, X., Zhang, W., Tao, M., Guo, Q., Wang, Z., \& Xia, A. (2019). Bridge-mediated charge separation in isomeric $\mathrm{N}$-annulated perylene diimide dimers. Journal of the American Chemical Society, 141, 12789-12796.

71. Ostroumov, E. E., Götze, J. P., Reus, M., Lambrev, P. H., \& Holzwarth, A. R. (2020). Characterization of fluorescent chlorophyll charge-transfer states as intermediates in the excited state quenching of light-harvesting complex II. Photosynthesis Research, 144, 171-193.

72. Connolly, J. S., Janzen, A. F., \& Samuel, E. B. (1982). Fluorescence lifetimes of chlorophyll a: Solvent, concentration and oxygen dependence. Photochemistry and Photobiology, 36, 559-563.

73. Gouterman, M. (1961). Spectra of porphyrins. Journal of Molecular Spectroscopy, 6, 138-163.

74. Abramczyk, H. (2012). Mechanisms of energy dissipation and ultrafast primary events in photostable systems: H-bond, excess electron, biological photoreceptors. Vibrational Spectroscopy, 58, $1-11$.

75. Rodriguez, J., Kirmaier, C., \& Holten, D. (1991). Time-resolved and static optical properties of vibrationally excited porphyrins. The Journal of Chemical Physics, 94, 6020-6029.

76. Nakanishi, T., Ohkubo, K., Kojima, T., \& Fukuzumi, S. (2009). Reorganization energies of diprotonated and saddle-distorted porphyrins in photoinduced electron-transfer reduction controlled 
by conformational distortion. Journal of the American Chemical Society, 131, 577-584.

77. Sazanovich, I. V., Galievsky, V. A., Van Hoek, A., Schaafsma, T. J., Malinovskii, V. L., Holten, D., \& Chirvony, V. S. (2001). Photophysical and structural properties of saddle-shaped free base porphyrins: Evidence for an 'orthogonal' dipole moment. The Journal of Physical Chemistry B, 105, 7818-7829.

78. Röder, B., Büchner, M., Rückmann, I., \& Senge, M. O. (2010). Correlation of photophysical parameters with macrocycle distortion in porphyrins with graded degree of saddle distortion. Photochemical \& Photobiological Sciences, 9, 1152-1158.
79. Gentemann, S., Medforth, C. J., Ema, T., Nelson, N. Y., Smith, K. M., Fajer, J., \& Holten, D. (1995). Unusual picosecond $1\left(\pi, \pi^{*}\right)$ deactivation of ruffled nonplanar porphyrins. Chemical Physics Letters, 245, 441-447.

80. Yin, S., Dahlbom, M. G., Canfield, P. J., Hush, N. S., Kobayashi, R., \& Reimers, J. R. (2007). Assignment of the Qy, absorption spectrum of photosystem-I from Thermosynechococcus elongatus based on CAM-B3LYP calculations at the PW91-optimized protein structure. The Journal of Physical Chemistry B, 111, 9923-9930. 